\title{
Genomic Characterization Reveals Insights Into Patulin Biosynthesis and Pathogenicity in Penicillium Species
}

\author{
Boqiang Li, ${ }^{1}$ Yuanyuan Zong, ${ }^{1}$ Zhenglin Du, ${ }^{2}$ Yong Chen, ${ }^{1}$ Zhanquan Zhang, ${ }^{1}$ Guozheng Qin, ${ }^{1}$ \\ Wenming Zhao, ${ }^{2}$ and Shiping Tian' ${ }^{1}$ \\ ${ }^{1}$ Key Laboratory of Plant Resources, Institute of Botany, Chinese Academy of Sciences, Beijing, China; ${ }^{2}$ Core Genomic Facility, \\ Beijing Institute of Genomics, Chinese Academy of Sciences
}

Submitted 2 October 2014. Accepted 22 January 2015.

\begin{abstract}
Penicillium species are fungal pathogens that infect crop plants worldwide. $P$. expansum differs from $P$. italicum and $P$. digitatum, all major postharvest pathogens of pome and citrus, in that the former is able to produce the mycotoxin patulin and has a broader host range. The molecular basis of host-specificity of fungal pathogens has now become the focus of recent research. The present report provides the whole genome sequence of $P$. expansum (33.52 $\mathrm{Mb})$ and $P$. italicum $(28.99 \mathrm{Mb})$ and identifies differences in genome structure, important pathogenic characters, and secondary metabolite (SM) gene clusters in Penicillium species. We identified a total of 55 gene clusters potentially related to secondary metabolism, including a cluster of 15 genes (named PePatA to $\mathrm{PePatO}$ ), that may be involved in patulin biosynthesis in P. expansum. Functional studies confirmed that PePatL and PePatK play crucial roles in the biosynthesis of patulin and that patulin production is not related to virulence of $P$. expansum. Collectively, $P$. expansum contains more pathogenic genes and SM gene clusters, in particular, an intact patulin cluster, than $P$. italicum or $P$. digitatum. These findings provide important information relevant to understanding the molecular network of patulin biosynthesis and mechanisms of host-specificity in Penicillium species.
\end{abstract}

Penicillium is a genus within ascomycetous fungi with great importance in the natural environment, as well as in food and drug production (Cheeseman et al. 2014; Fierro et al. 1995). Several species, such as $P$. expansum, $P$. italicum, and $P$. digitatum, are wellknown postharvest pathogens that are responsible for losses of up to $10 \%$ of harvested produce. P. expansum can infect a wide range of fruit and vegetable hosts, except citrus fruit, and produces the mycotoxin patulin (Andersen et al. 2004). In contrast, $P$. digitatum and $P$. italicum are restricted to citrus fruit and do not produce patulin. Patulin is a tetraketide mycotoxin that can induce immunological, neurological, and gastrointestinal diseases leading to serious health

\section{B. Li and Y. Zong contributed equally to this work.}

Genome assembly data is available in the DDBJ/EMBL/GenBank database under accessions AYHP00000000 (P. expansum) and JMDK00000000 (P. italicum). RNA-sequencing data is deposited in Sequence Read Archive (SRA) under project number SRP043647 (accession numbers SRX647239, SRX647240, SRX647241, SRX647242).

Corresponding author: S. Tian: E-mail: tsp@ibcas.ac.cn; Telephone: +8610-6283-6559; Fax: +86-10-8259-4675.

*The $e$-Xtra logo stands for "electronic extra" and indicates that four supplementary figures and 11 supplementary tables are published online.

(c) 2015 The American Phytopathological Society problems in humans (Bennett and Klich 2003; Pfeiffer et al. 1998). The biosynthetic pathway of patulin contains at least ten enzymatic steps (Moake et al. 2005). A few genes associated with patulin biosynthesis, such as 6-msas encoding a 6-methylsalicylic acid synthase, $i d h$ encoding an isoepoxydon dehydrogenase, and peabl encoding an ATP-binding cassette transporter, have been cloned from Penicillium species (Wang et al. 1991; White et al. 2006). In addition, a 15-gene patulin cluster was reported in Aspergillus clavatus (Artigot et al. 2009) and P. expansum (Tannous et al. 2014). Fungal phytotoxins have been shown to act as a mediator of pathogenicity or virulence in several mycotoxigenic genera that infect many agricultural crops and cause significant disease (Möbius and Hertweck 2009; Yoder and Turgeon 1996). Whether or not mycotoxin biosynthesis directly contributes to fungal pathogenicity, however, remains an open question in many host-pathogen systems. Therefore, exploring the molecular basis of pathogenicity and patulin biosynthesis in Penicillium species is biologically interesting and may have economic significance.

Previous studies have indicated that signaling pathways that connect fungal development with mycotoxin biosynthesis would be scattered throughout the genome (Andersen et al. 2013; Fierro et al. 1995; van den Berg et al. 2008). However, the complexity of these regulatory networks and their interconnection with other regulatory mechanisms remains to be discovered. Comparative genomics is a tool that can be used to develop a better understanding of the molecular basis of fungal pathogenicity and patulin biosynthesis in Penicillium species. Here, we provide and report two high-quality draft genomes: a $33.52-\mathrm{Mb} P$. expansum genome containing 11,770 predicted protein-coding genes and a 28.99-Mb P. italicum genome containing 9,369 predicted protein-coding genes. The genome structure of both species was comprehensively analyzed and important pathogenic characters and secondary metabolite (SM) gene clusters in all three Penicillium species were examined. Notably, $P$. expansum contains more carbohydrate active enzymes (CAZymes) and a larger secretome as well as more SM gene clusters than P. italicum and $P$. digitatum. We also identified a cluster of 15 genes potentially related to patulin biosynthetic pathway of $P$. expansum. Our findings lay the foundation for further understanding the molecular basis of pathogenicity and patulin biosynthesis in Penicillium species.

\section{RESULTS}

Genome sequencing, assembly, and characterization.

Two Penicillium species, $P$. expansum T01 and P. italicum B3 were sequenced using a whole-genome shotgun-sequencing strategy. The assembled genome of $P$. expansum is $33.52 \mathrm{Mb}$ 
(Fig. 1; Table 1), and the total length of the eight largest scaffolds is $32.9 \mathrm{Mb}$ (approximately $98 \%$ of the assembled genome). A highquality draft genome $(28.99 \mathrm{Mb})$ of $P$. italicum B3 was also obtained. The genome of both species is slightly larger than the $26 \mathrm{Mb}$ reported for the related species $P$. digitatum (Marcet-Houben et al. 2012). Repetitive elements comprise $1.78 \%$ of the $P$. expansum genome and $1.8 \%$ of the P. italicum genome (Table 1). Repetitive elements are clustered in genomic regions with a low GC content (Fig. 1C and D), which is similar to reports in Colletotrichum graminicola (O'Connell et al. 2012) and Leptosphaeria maculans (Rouxel et al. 2011). There is a high colinearity between the genomes of P. expansum and P. italicum (Fig. 1E). A total of 11,770 and 9,369 protein-coding genes were predicted from the genome sequences of $P$. expansum and P. italicum, respectively. The two Penicillium species also shared a similar profile of Gene Ontology (GO) categories, but the number of proteins in each functional category is greater in $P$. expansum than in P. italicum (Supplementary Fig. S1).

\section{Comparative genomics of three}

Penicillium phytopathogens.

Based on a phylogenetic analysis, $P$. expansum is more closely related to $P$. italicum than to $P$. digitatum (Fig. 2A). The three Penicillium species share 6,222 gene families (Fig. 2B), using Markov cluster (MCL) analysis (Enright et al. 2002). In addition to the core gene families that are present in all three species, $P$. expansum shares more gene families (898) with $P$. italicum than $P$. digitatum (224). Notably, 102 gene families are uniquely shared by $P$. italicum and $P$. digitatum. The shared and specific gene families in the three Penicillium species were

Table 1. Assembly and genome characteristics of Penicillium expansum T01 and P. italicum B3

\begin{tabular}{lcc}
\hline Genome features & P. expansum T01 & P. italicum B3 \\
\hline Genome size $(\mathrm{Mb})$ & 33.52 & 28.99 \\
Sequencing coverage & $527 \times$ & $193 \times$ \\
Number of contigs & 4,294 & 4,873 \\
$\mathrm{~N}_{50}$ contig (Kb) & 123.62 & 161.33 \\
Number of scaffolds ( $>500 \mathrm{bp})$ & 108 & 822 \\
Number of scaffolds (>1 kb) & 36 & 640 \\
Max scaffold length (kb) & $9,171.49$ & 829.49 \\
$\mathrm{~N}_{50}$ scaffold (Kb) & $5,031.09$ & 205.92 \\
GC content $(\%)$ & 47.10 & 47.03 \\
Protein-coding genes & 1,1770 & 9,369 \\
Mean gene length (bp) & 1,891 & 1,648 \\
Mean CDS length (bp) & 1,464 & 1,481 \\
Mean exons per gene & 3 & 3 \\
Mean exons length (bp) & 486 & 490 \\
Mean intron length (bp) & 80 & 82 \\
Min protein length (aa) & 50 & 50 \\
Max protein length (aa) & 7,257 & 7,288 \\
Repetitive elements (\%) & 1.78 & 1.80 \\
\hline
\end{tabular}

A
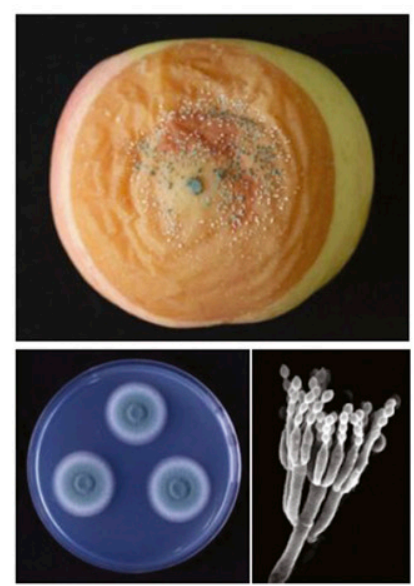

C

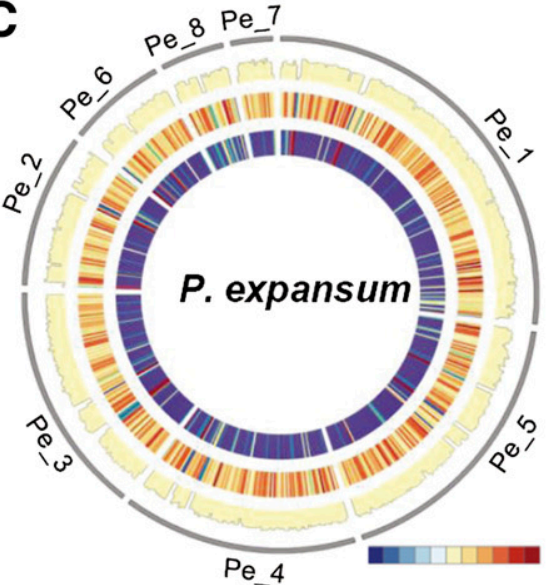

B

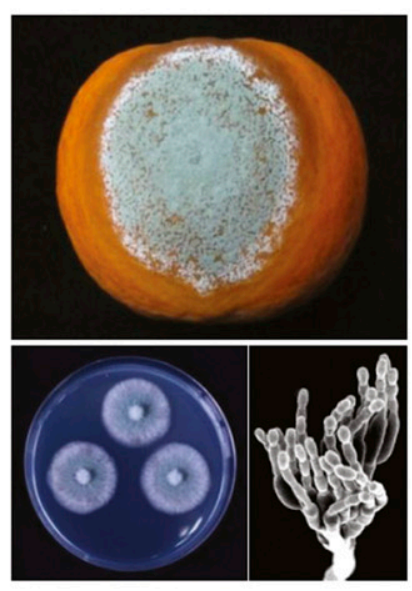

D

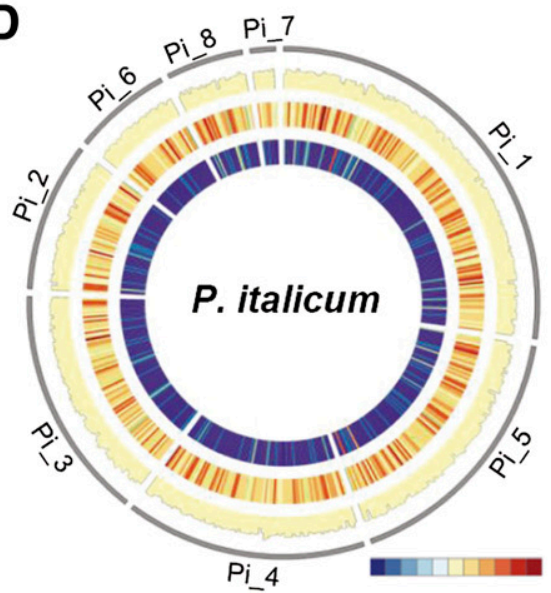

E

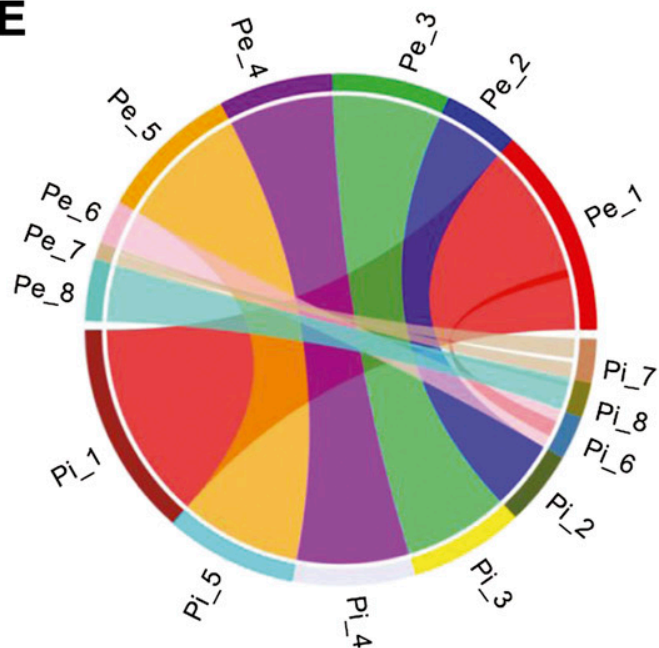

Fig. 1. Genomes of Penicillium expansum and P. italicum. A, Colony, microscopic morphology of $P$. expansum, and typical disease symptoms in apple fruit. B, Colony, microscopic morphology of $P$. italicum, and typical disease symptoms in orange fruit. $\mathbf{C}$ and $\mathbf{D}$, Genome organization and gene distribution in $P$. expansum and P. italicum. The peripheral circles represent the scaffolds. Pe 1 to Pe 8 represent the longest eight scaffolds of $P$. expansum. The $P$. italicum genome was aligned to these eight scaffolds. Based on the results of the alignment, $P$. italicum scaffold sequences were merged together and designated as Pi_1 to Pi_8. The second circles indicate the GC content with the ratio of GC sites per $50 \mathrm{~kb}$ ranged from 0.35 to 0.53 . The third circles indicate the gene density with the frequency of sites per $50 \mathrm{~kb}$ ranged from 1 to 28 . The fourth circles indicate the distribution of repetitive DNA elements with the frequency of sites per $50 \mathrm{~kb}$ ranged from 0 to 0.1 . E, Genome synteny map between $P$. expansum and $P$. italicum. 
classified by GO analysis according to biological processes (Fig. 2C). The majority of the 6,222 core families (Circle I) were classified into the functional categories of cellular $(2,160$ families) and metabolic (2,554 families) processes. Approximately half of the species-specific gene families (Circles $\mathrm{V}$ to VII) could not be assigned into a designated GO category (Fig. 2C). MCL analysis revealed that the three Penicillium species share similar multimember gene families, including a major facilitator superfamily (MFS) of transporters, amino acid permeases, and betaine aldehyde dehydrogenases (Supplementary Table S1). P. expansum has more members in these gene families than $P$. italicum and $P$. digitatum.

Using the CAZymes analysis toolkit (CAT) (Park et al. 2010), 640, 460, and 394 putative CAZymes were identified in $P$. expansum, $P$. italicum, and $P$. digitatum, respectively (Fig. 3A; Supplementary Table S2). P. expansum was found to possess a greater number of carbohydrate esterases (CE), glycoside hydrolases (GH), and polysaccharide lyases (PL) and a similar number of glycosyltransferases (GT) relative to $P$. italicum and $P$. digitatum (Fig. 3A). The greater number of $\mathrm{CE}, \mathrm{GH}$, and $\mathrm{PL}$ in $P$. expansum may be due to the larger genome size of $P$. expansum relative to the other two species. CE, GH, and PL are associated with the ability to utilize the diversity of carbohydrates present in the environment and within host plants.
GT are mainly involved in the basal activities of fungal cells (e.g., fungal cell-wall synthesis, glycogen cycle, trehalose cycle), and so the number and type of GT tend to be conserved in fungi (Lombard et al. 2014; O'Connell et al. 2012). It is well known that CAZymes involved in the degradation of the plant cell wall are important for the pathogenicity of plant pathogens. All three Penicillium species were found to encode a smaller number of cellulose-degrading enzymes (less than 10 proteins [Fig. 3B]) compared with hemicellulose- and pectin-degrading enzymes (Fig. 3C, D, and E).

Pectin is abundant in fruit cell walls, and apple and citrus are the main raw materials used for the industrial production of pectin. The three Penicillium species have genes encoding proteins in nine of the pectin-targeting CAZyme families, including three PL families (PL1, PL3, and PL4), four GH families (GH28, GH78, GH95, and GH105), and two CE families (CE8 and CE12). In general, the three Penicillium species have a similar number of representatives in each of above pectin-targeting CAZyme families except in the GH78 family. P. expansum has eight genes encoding for GH78 family enzymes, which is twice that of $P$. italicum (three enzymes) and $P$. digitatum (four enzymes) (Fig. 3D). Pectin is composed of homogalacturonans ("smooth" regions) and more complex rhamnogalacturonans that contain arabinan, galactan, and
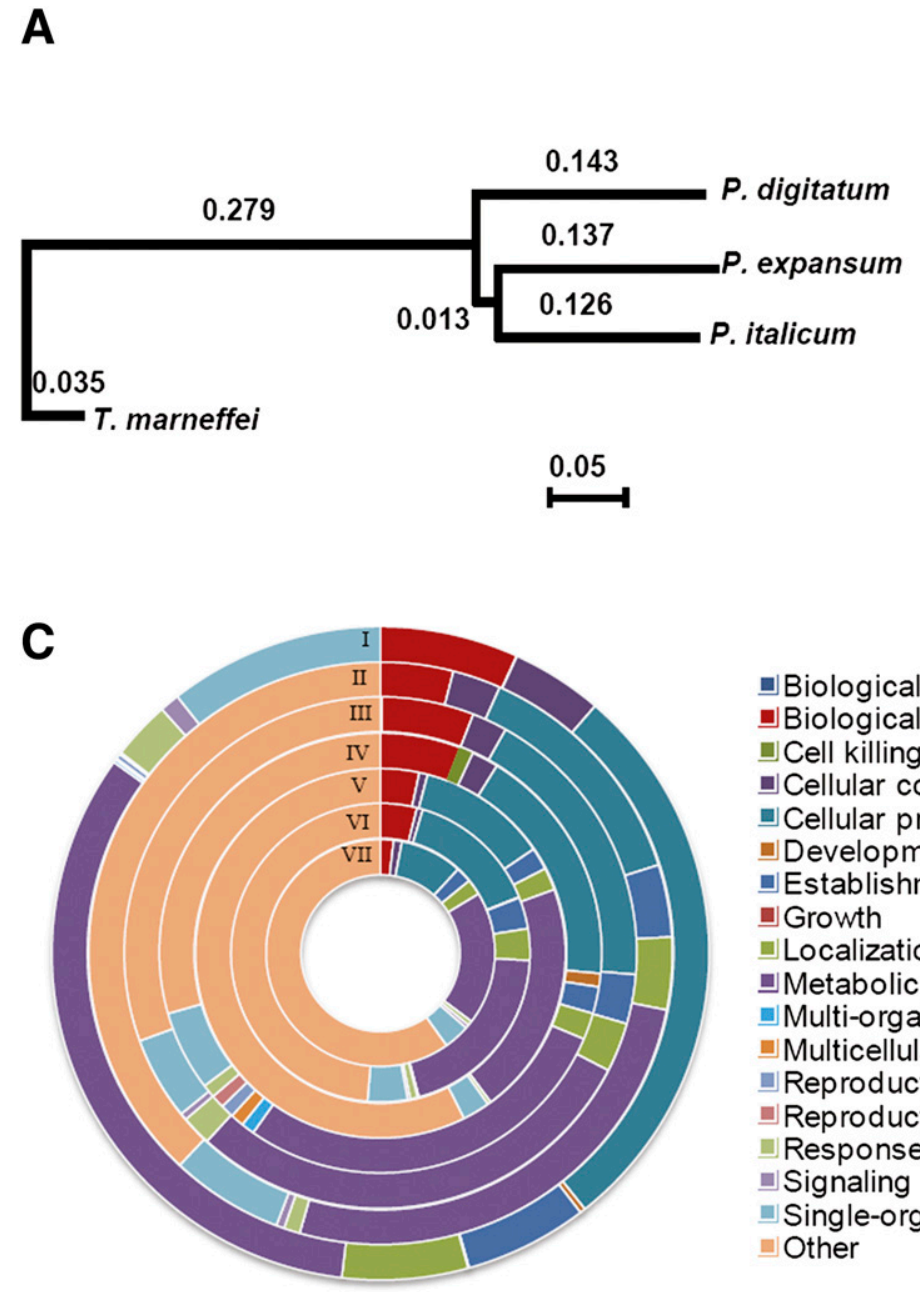

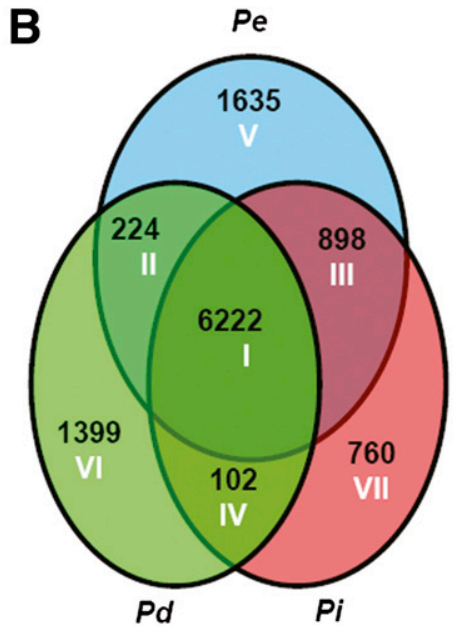

- Biological adhesion - Biological regulation - Cell killing - Cellular component organization or biogenesis - Cellular process

- Developmental process

Establishment of localization

$\square$ Growth

- Localization

- Metabolic process

- Multi-organism process

- Multicellular organismal process

- Reproduction

- Reproductive process

- Response to stimulus

- Signaling

- Single-organism process

- Other

Fig. 2. Genome comparison between Penicillium expansum, P. italicum, and P. digitatum. A, A whole-genome cladogram showing the phylogenetic relationships between the three Penicillium species was constructed using CVTree with Talaromyces marneffei as an outgroup. Branch lengths corresponding to substitutions per site were calculated using a maximum likelihood approach. B, A Venn diagram showing the number of shared and specific gene families. $P e=$ Penicillium expansum, $P i=P$. italicum, and $P d=P$. digitatum. C, Gene Ontology $(\mathrm{GO})$ categories of gene families according to biological process. Circles I to VII correspond to areas I to VII in B. 
arabinogalactan side chains ("hairy" regions). GH78 enzymes exhibit $\alpha$-L-rhamnosidase activity and are involved in the degradation of rhamnogalacturonan backbone of the hairy region in pectin molecules. The greater number of genes in $P$. expansum encoding for GH78 family enzymes may represent an adaption to the structure of apple pectin, which has a high proportion of hairy regions (Wang et al. 2014). The expression of eight GH78 family genes in P. expansum when it was supplied with apple or citrus pectin as a carbon source was examined (Fig. 3G). Expression of all eight GH78 family genes increased with time of culture when either apple or citrus pectin was used. Relative expression of four of the genes (PEG03271, PEG05990, PEG10044, and PEG11722) was two- to sixfold greater after $48 \mathrm{~h}$ when $P$. expansum was cultured on apple pectin rather than citrus pectin, suggesting that those genes may play an important role in the degradation of the apple pectin. Besides rhamnogalacturonan backbone, the hairy region has one or both arabinan and arabinogalactan-rich side chains substituted onto rhamnose residues (Blackman et al. 2014). Wang et al. (2014) reported that the structure of arabinogalactan side chains in apple and citrus pectin are different. A total of 17, 13, and eight CAZymes were identified in $P$. expansum, $P$. italicum, and $P$. digitatum, respectively, that may function in the degradation of side chains in the hairy region (Fig. 3E). Since CAZymes in GH43, GH51, GH53, GH54, and GH93 families can also degrade hemicelluloses and other substrates, it is difficult to specifically identify the number of GH involved in the degradation of pectin side chains (Amselem et al. 2011; Blackman et al. 2014).
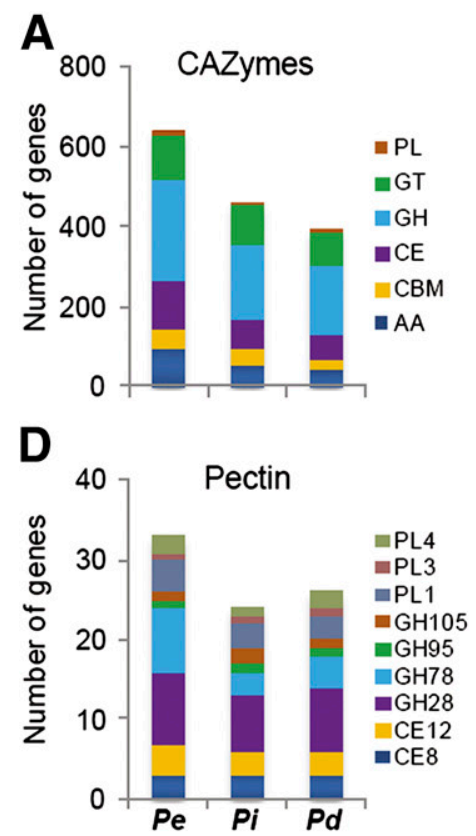

B

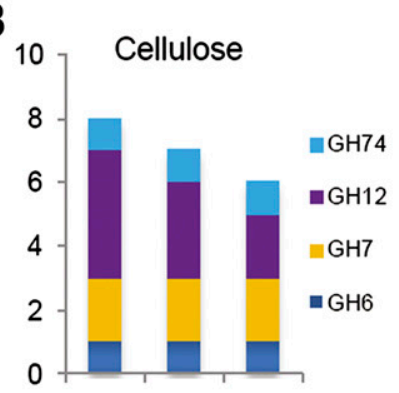

E

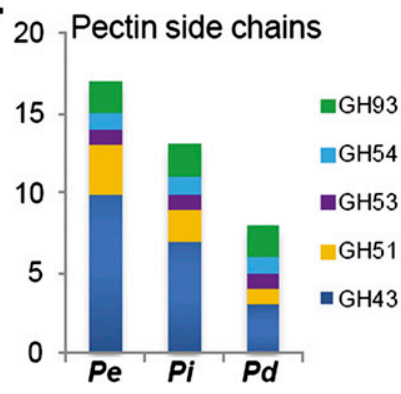

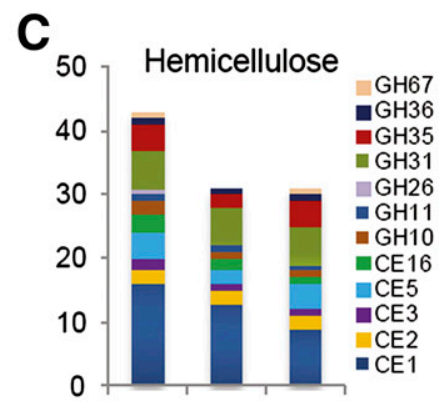

$\mathbf{F}$

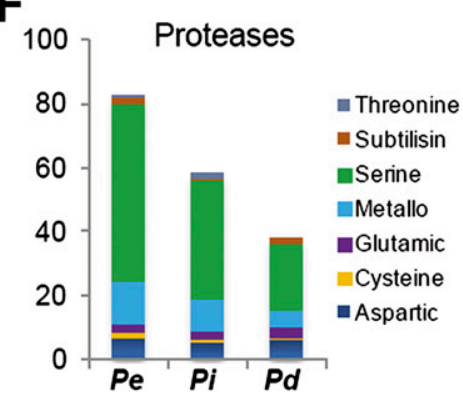

G $\quad$ Citrus pectin $=$ Apple pectin

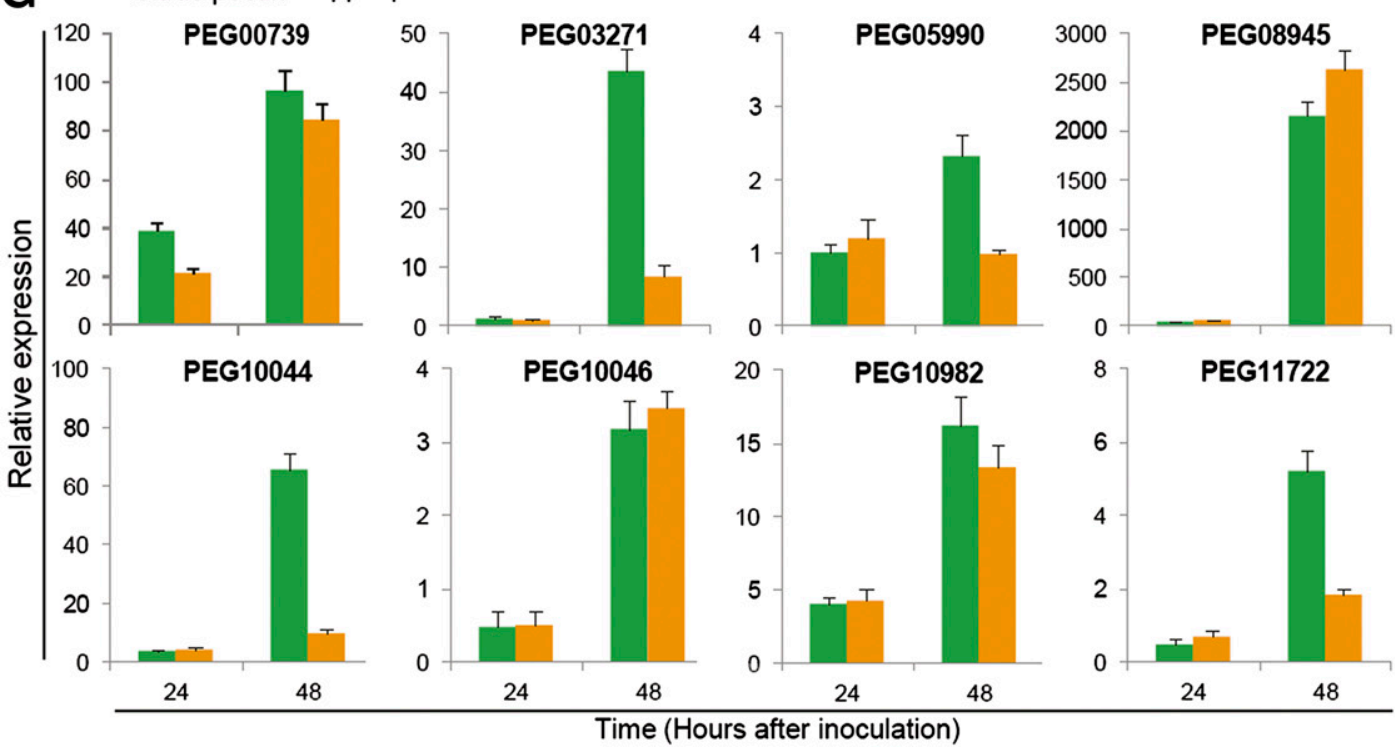

Fig. 3. Comparison of genes encoding carbohydrate active enzymes (CAZymes) and secreted proteases in Penicillium expansum, P. italicum, and P. digitatum. A, CAZymes identified in the genome of $P$. expansum, $P$. italicum, and $P$. digitatum . PL $=$ polysaccharide lyase, GT $=$ glycosyltransferase, GH $=$ glycoside hydrolase, $\mathrm{CE}=$ carbohydrate esterase, $\mathrm{CBM}=$ carbohydrate-binding module, and AA = auxiliary activity. $\mathbf{B}, \mathrm{CAZymes}$ involved in cellulose degradation, $\mathbf{C}$, hemicellulose degradation, $\mathbf{D}$, pectin degradation, and $\mathbf{E}$, degradation of pectin side chains. F, Secreted proteases. $P e=P e n i c i l l i u m$ expansum, $P i=P$. italicum, and $P d=P$. digitatum. G, Reverse transcription quantitative polymerase chain reaction analysis of genes encoding GH78 family enzymes in $P$. expansum when cultured with either apple or citrus pectin as a carbon source. 
P. expansum also possesses a larger secretome ( 927 predicted secreted proteins, $7.7 \%$ of the proteome) than $P$. italicum (662, $7.1 \%)$ and $P$. digitatum $(546,6.0 \%)(P<0.05$ according to Fisher's exact test). Proteases are abundant in the secretome of all species, with $P$. expansum containing over twice as many secreted proteases as $P$. digitatum (Fig. 3F). Possessing a diversity of secreted proteases is beneficial, since it allows the fungus to exploit a variety of ambient nutrients and also to counteract protein-based defense responses of the hosts. As shown in Figure 3F, more than half of the identified secreted proteases belong to a superfamily of serine proteases. Among them, the $\mathrm{S} 33$ family is lacking in $P$. italicum and $P$. digitatum while $P$. expansum encodes three S33 proteases (Supplementary Table S3). S33 proteases exhibit prolyl aminopeptidase activity, which releases $\mathrm{N}$-terminal proline from a peptide. P. expansum also has some unique families, such as M6, M12B, M20D, M35, and M77, that belong to a superfamily of metallo proteases.

\section{Identification of gene clusters related} to secondary metabolism and patulin biosynthesis.

Typically, a SM gene cluster contains one or more "backbone" genes that encode key enzymes of the SM biosynthetic process, such as polyketide synthase (PKS), nonribosomal peptide synthetase (NRPS), a PKS-NRPS hybrid, dimethylallyl tryptophan synthase (DMATS). Using the bioinformatic program SMURF (Khaldi et al. 2010), 71 predicted SM backbone genes were found to be present in $P$. expansum (Supplementary Table S4), which is distinctly more than were found in P. italicum (38 backbone genes) and P. digitatum (32 backbone genes) (Fig. 4A). Phylogenetic analysis indicated that some of the SM backbone genes present in these three species clustered together (Fig. 4B). In addition to possessing an increased number of SM backbone genes, $P$. expansum also contains the largest number of cytochrome P450 (CYP450)
(Fig. 4C) and transporter (Fig. 4D) genes, both of which play important roles in the biosynthesis and transportation of SM (Del Sorbo et al. 2000; Weng et al. 2012). Among the transporter genes, $P$. expansum contains a larger number of MFS transporters $(527,54 \%$ of total transporters) than $P$. italicum (386, 42\%) and P. digitatum $(258,41 \%)$ (Fig. 4D). In contrast, a smaller difference in the number of $\mathrm{ABC}$ transporter genes exists between the three Penicillium species.

In total, $55 \mathrm{SM}$ clusters were identified in $P$. expansum as compared with $30 \mathrm{SM}$ clusters in P. italicum and 24 clusters in $P$. digitatum (Supplementary Table S5). Some SM clusters are shared by all three or by two of the Penicillium species (Fig. 5A and B), indicating that the three Penicillium species may produce some similar SM. Only $P$. expansum, however, possesses the complete patulin gene cluster (Fig. 5B). This 44-kb cluster consists of 15 genes, named PePatA to PePatO. Each of the genes in the patulin cluster of $P$. expansum shares high identity with a corresponding gene in the putative patulin cluster of A. clavatus (Table 2), but the order of the genes within the cluster is quite different in the two species (Fig. 5C). Among the 15 genes in the patulin cluster of $P$. expansum, one encodes a putative transcription factor (PePatL), three encode transporters (PePatA, PePatC, and PePatM), nine encode biosynthetic enzymes, and two (PePatF and PePatJ) have an unknown function (Table 2). PePatF has a SnoaL-like domain, and PePatJ has a Cupin domain based on a Pfam analysis. Proteins with those domains commonly exhibit enzymatic activity in microorganisms (Dunwell et al. 2000; Sultana et al. 2004). PePatK is the backbone gene of the patulin cluster in $P$. expansum. It encodes a 6-methylsalicylic acid synthase (6-MSAS), which belongs to the type I PKS group and has five different domains (Supplementary Fig. S2). Although a few orthologs of patulin genes were also found in $P$. italicum and $P$. digitatum, the backbone gene $(P a t K)$ was absent in both
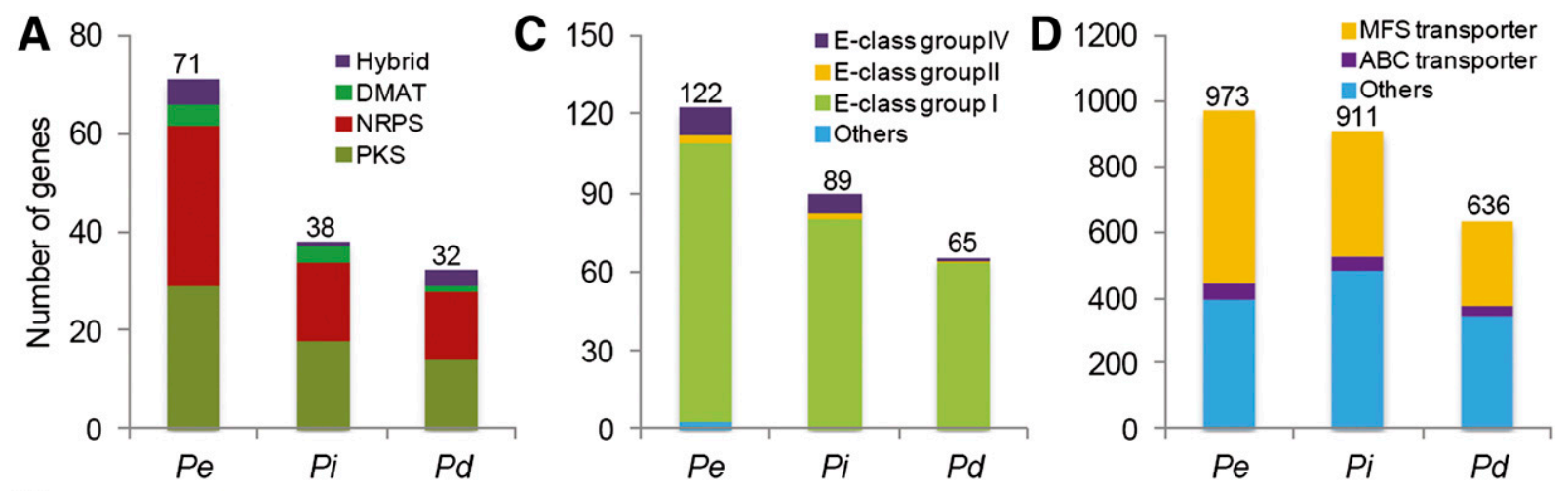

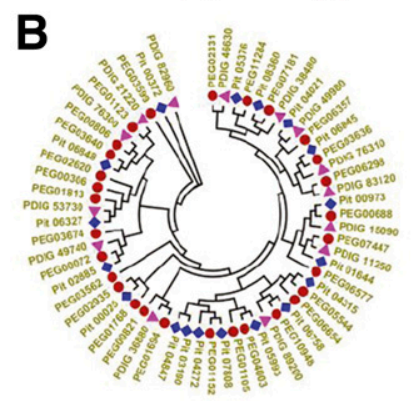

PKS
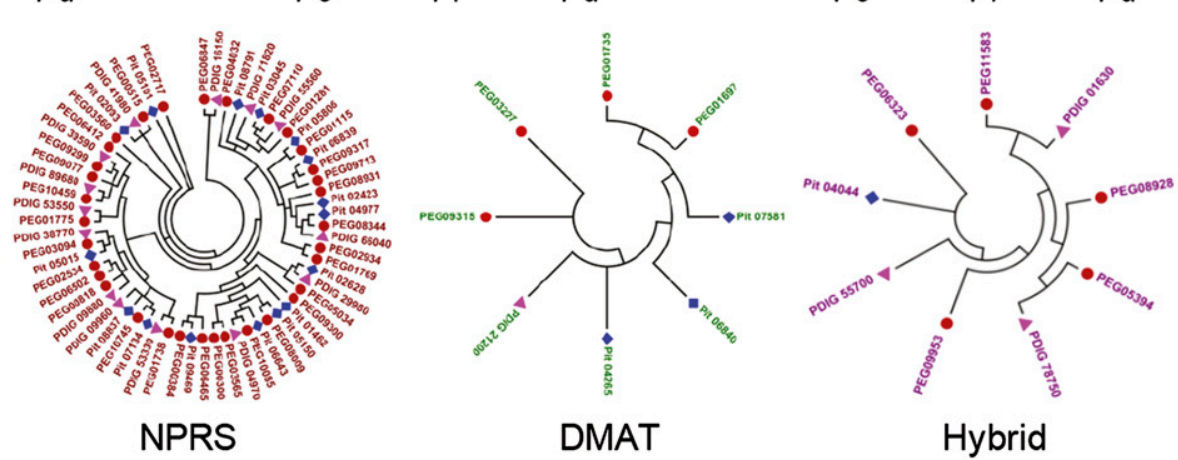

Fig. 4. Comparison of genes involved in secondary metabolism in Penicillium expansum, P. italicum, and P. digitatum. A, Secondary metabolite backbone genes. $P e=$ Penicillium expansum, $P i=P$. italicum, $P d=P$. digitatum; PKS =polyketide synthase; NRPS $=$ nonribosomal peptide synthetase; and DMATS $=$ dimethylallyl tryptophan synthase. B, Phylogenetic analysis of genes encoding PKS, NRPS, PKS-NRPS hybrids, and DMATS in three Penicillium species. Dots indicate backbone genes of Penicillium expansum; diamonds indicate backbone genes of $P$. italicum; and triangles indicate backbone genes of P. digitatum. C, Genes encoding CYP450s. D, Genes encoding transporters. 
and only three patulin genes (PatC, PatD, and PatL) were identified in P. italicum (Fig. 5C). PatC and PatD appeared to fuse into one predicted gene (Pit_00308) in P. italicum. The loss of the backbone and other patulin genes negates the ability to produce patulin in $P$. italicum and $P$. digitatum.

\section{Expression analysis of genes involved} in patulin biosynthesis.

The transcriptome of $P$. expansum cultured in static (patulin permissive) and shaking (patulin restrictive) conditions were compared in order to investigate the expression of genes potentially related to patulin biosynthesis. Approximately 3,034 genes were differentially expressed $(P \leq 0.001)$ in both conditions (Fig. 6A) and, based on GO functional classification, most could be assigned to catalytic activity and metabolic process (Supplementary Fig. S3). Among the differentially expressed genes, 1,642 were up-regulated under static conditions, including all 15 genes in the patulin cluster, and 1,392 genes were down-regulated (Fig. 6A).

Differentially expressed genes were enriched in the ko03010 (ribosome) pathway based on KEGG analysis (Supplementary Tables S6 and S7), and the ortholog of LaeA (PEG09421) was significantly up-regulated in the static culture (Supplementary Table S8). LaeA is a well-known regulator of secondary metabolism in A. nidulans (Bok and Keller 2004). This protein is thought to activate expression of SM gene clusters by chromatin modification mediated by the velvet complex (Bayram et al. 2008; Strauss and Reyes-Dominguez 2011). Differential expression of the SM backbone genes and genes encoding CYP450s, ATP-binding cassette (ABC) transporters, and MFS
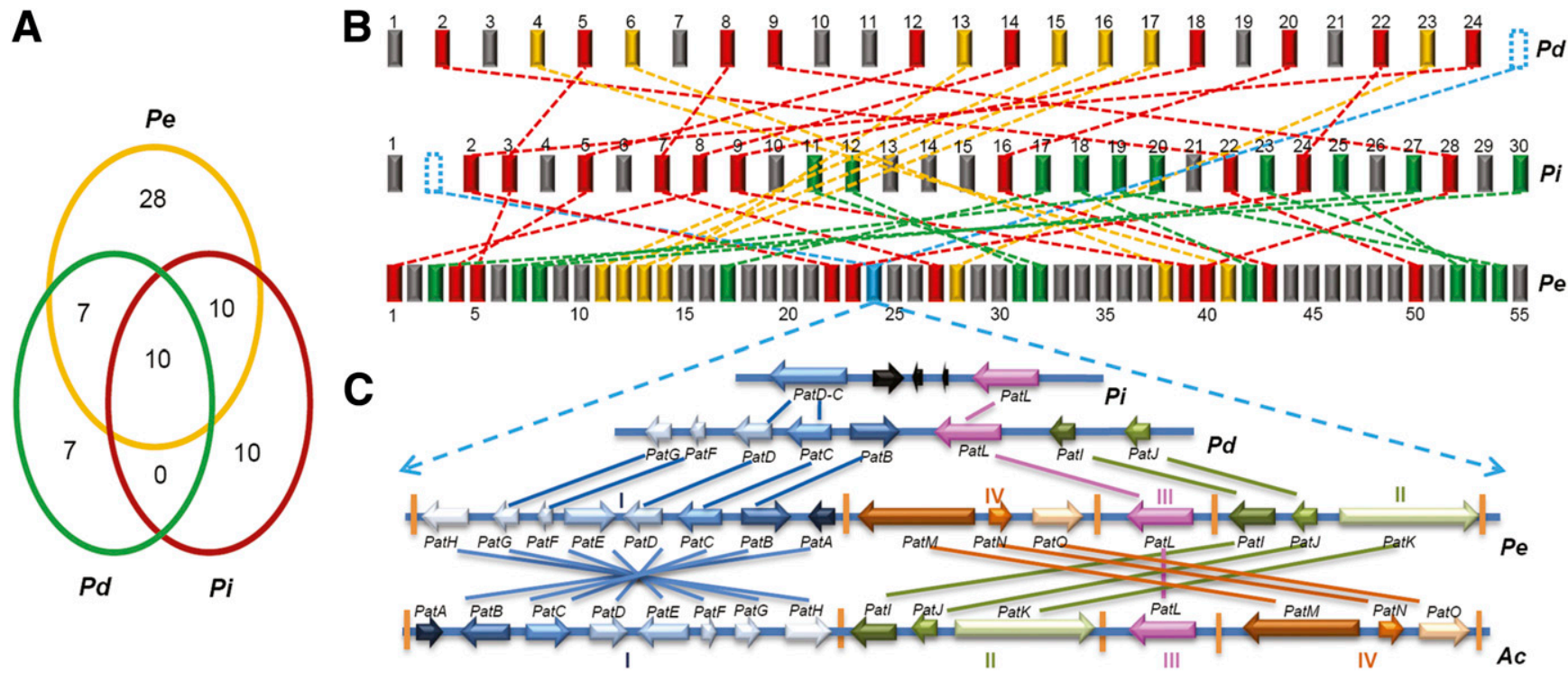

Fig. 5. Comparison of secondary metabolite gene clusters and structure of the patulin gene clusters. A, A Venn diagram shows the number of shared secondary metabolite gene clusters in Penicillium expansum (Pe), P. italicum (Pi), and P. digitatum $(P d)$. B, Clusters shared by three Penicillium species. Red solid bars: clusters shared by $P$. expansum, $P$. italicum, and $P$. digitatum; green solid bars: clusters shared by $P$. expansum and $P$. italicum; yellow solid bars: clusters shared by $P$. expansum and $P$. digitatum; gray solid bars: specific clusters in each Penicillium species; blue solid bar: the patulin cluster in $P$. expansum; blue hollow bars: the incomplete patulin clusters in P. italicum, and P. digitatum. C, Structure comparison of patulin gene clusters in three Penicillium species and Aspergillus clavatus $(A c)$. Black arrows represent genes not involved in patulin biosynthesis. Patulin clusters in $P$. expansum and A. clavatus were artificially divided into four regions (I to IV) separated by orange vertical lines.

Table 2. Comparison of genes in the patulin clusters of Aspergillus clavatus (Ac) and Penicillium expansum $(P e)^{\mathrm{a}}$

\begin{tabular}{|c|c|c|c|c|c|c|c|c|c|}
\hline \multirow[b]{2}{*}{ Gene } & \multicolumn{2}{|c|}{ Indentification number } & \multirow[b]{2}{*}{ Putative function } & \multicolumn{2}{|c|}{$\begin{array}{l}\text { Length of } \\
\text { open reading } \\
\text { frame (bp) }\end{array}$} & \multicolumn{2}{|c|}{ Exon } & \multirow{2}{*}{$\begin{array}{c}\text { Nucleotide } \\
\text { identity }(\%)\end{array}$} & \multirow{2}{*}{$\begin{array}{c}\text { Protein } \\
\text { identity }(\%)\end{array}$} \\
\hline & $A c$ & $P e$ & & $A c$ & $P e$ & $A c$ & $P e$ & & \\
\hline PatA & ACLA_093560 & PEG03683 & Acetate transporter & 1,007 & 1,041 & 5 & 5 & 63.05 & 78.09 \\
\hline PatB & ACLA_093570 & PEG03684 & Carboxylesterase & 1,872 & 1,927 & 4 & 4 & 63.09 & 67.2 \\
\hline $\mathrm{PatC}$ & ACLA_093580 & PEG03685 & MFS transporter & 1,781 & 1,752 & 3 & 3 & 72.18 & 77.41 \\
\hline$P a t D$ & ACLA_093590 & PEG03686 & Alcohol dehydrogenase & 1,491 & 1,511 & 5 & 7 & 63.65 & 73.2 \\
\hline PatE & ACLA_093600 & PEG03687 & Glucose-methanol-choline oxidoreductase & 2,013 & 2,001 & 3 & 3 & 70.32 & 79.17 \\
\hline PatF & ACLA_093610 & PEG03688 & Hypothetical protein 1 & 597 & 600 & 1 & 1 & 71.50 & 77 \\
\hline PatG & ACLA_093620 & PEG03689 & Amidohydrolase family protein & 1,061 & 1,025 & 2 & 2 & 68.33 & 78.46 \\
\hline $\mathrm{PatH}$ & ACLA_093630 & PEG03690 & m-Cresol methyl hydroxylase & 1,854 & 1,841 & 5 & 5 & 70.80 & 84.54 \\
\hline PatI & ACLA_093640 & PEG03676 & m-Hydroxybenzyl alcohol hydroxylase & 1,926 & 1,886 & 5 & 5 & 67.38 & 80.41 \\
\hline PatJ & ACLA_093650 & PEG03675 & Hypothetical protein 2 & 1,117 & 1,116 & 3 & 3 & 70.38 & 84.36 \\
\hline PatK & ACLA_093660 & PEG03674 & 6-Methylsalicylic acid synthase & 5,163 & 5,464 & 1 & 2 & 68.48 & 77.8 \\
\hline PatL & ACLA_093670 & PEG03677 & C6 transcription factor & 2,541 & 2,456 & 2 & 2 & 64.67 & 66.87 \\
\hline PatM & ACLA_093680 & PEG03681 & $\mathrm{ABC}$ transporter & 4,630 & 4,553 & 7 & 7 & 68.95 & 76.69 \\
\hline PatN & ACLA_093690 & PEG03680 & Isoepoxydon dehydrogenase & 908 & 896 & 3 & 3 & 74.40 & 90.35 \\
\hline $\mathrm{PatO}$ & ACLA_093700 & PEG03678 & Isoamyl alcohol oxidase & 1,973 & 1,960 & 5 & 5 & 65.47 & 71.68 \\
\hline
\end{tabular}

${ }^{\mathrm{a}} \mathrm{MFS}=$ major facilitator superfamily, $\mathrm{ABC}=\mathrm{ATP}$-binding cassette, $\mathrm{ORF}=$ open reading frame . 
transporters were also observed (Fig. 6B). Approximately half the SM backbone genes were differentially expressed, including 15 genes encoding PKS, 16 genes encoding NRPS, four genes encoding PKS-NPRS hybrids, and two genes encoding DMAT (Fig. 6B). Among these genes, 23 were upregulated and 14 were down-regulated under static conditions. In addition, 12 genes encoding $\mathrm{ABC}$ transporters were upregulated and two were down-regulated. Reverse transcription quantitative polymerase chain reaction (RT-qPCR) analysis confirmed that all of the genes in the patulin cluster were upregulated when $P$. expansum was grown under static conditions (Fig. 6C) that are conducive to patulin production (Fig. 6D).

\section{Relationship Between patulin production} and pathogenicity in $P$. expansum.

To explore the role of patulin in the pathogenesis of $P$. expansum, two genes in the patulin cluster, PePatK and PePatL, were deleted, using a gene replacement strategy and Agrobacteriummediated transformation (Fig. 7A). PePatK encodes a putative
PKS and PePatL encodes a putative transcription factor. These two genes were selected as the target genes for the knockout assay because they were predicted to play an important role in the biosynthesis of patulin in $P$. expansum. Three knockout mutants were obtained for each target gene identified by PCR (Supplementary Fig. S4) and confirmed by Southern blot analysis (Fig. 7B). None of the knockout mutants of PePatL and PePatK produced detectable patulin as determined by highperformance liquid chromatography (HPLC) analysis (Fig. 7D). The knockout mutants of PePatL and PePatK exhibited a slightly smaller colony diameter, relative to the growth of the wild-type strain (Fig. 7E). No significant difference in sporulation was observed, however, between the knockout mutants and the wild-type strain (Fig. 7F). Two pome fruit hosts (apple and pear) and one stone fruit host (plum) were used in a pathogenicity assay to investigate the effect of patulin production on the pathogenicity in P. expansum. Results indicated that there was no significant difference in pathogenicity between the knockout mutants and the wild-type strain on any of three fruit hosts (Fig. 7G).
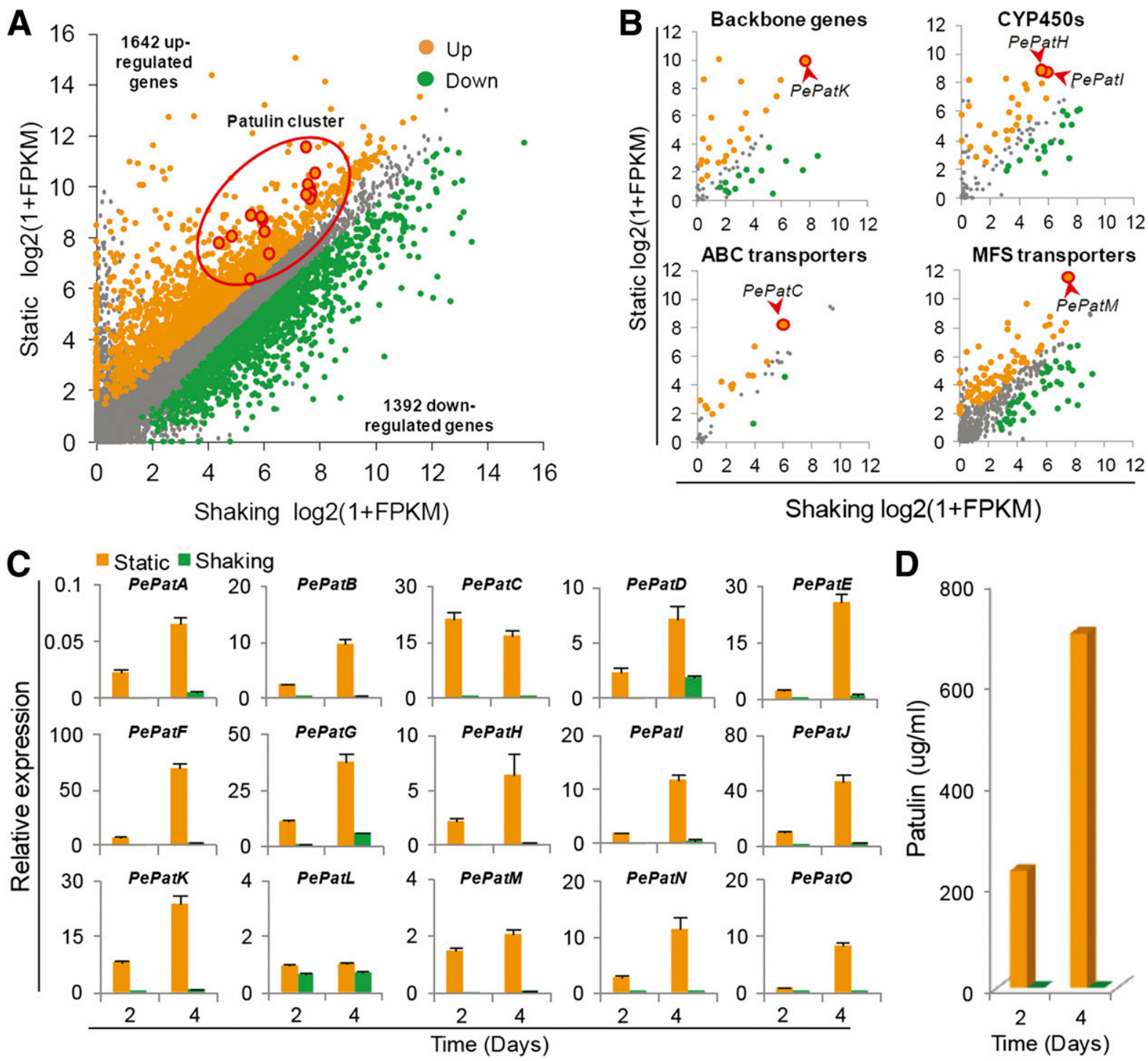

Fig. 6. Analysis of gene expression in Penicillium expansum cultured under patulin permissive (static) and restrictive (shaking) conditions. A, Scatterplot showing significantly up- and downregulated genes as revealed by transcriptome analysis. All of the genes in the patulin cluster (dots with dark borders) were up-regulated. B, Differential expressions of genes involved in secondary metabolism. Arrowheads point to genes included in the patulin cluster. C, Reverse transcription quantitative polymerase chain reaction analysis of the expression of patulin cluster genes after 2 and 4 days of static or shaking culture conditions. D, Patulin production after 2 and 4 days of static or shaking culture conditions. 
Characterization of PePatL, the putative transcription factor.

A common feature of fungal gene clusters responsible for the production of SM is the presence of a pathway-specific regulatory gene (Yu and Keller 2005; Yu et al. 1996). PePatL encodes the only putative transcription factor in the patulin cluster. The amino-acid sequence of PePatL was compared with several known transcription factors, such as GAL4 in
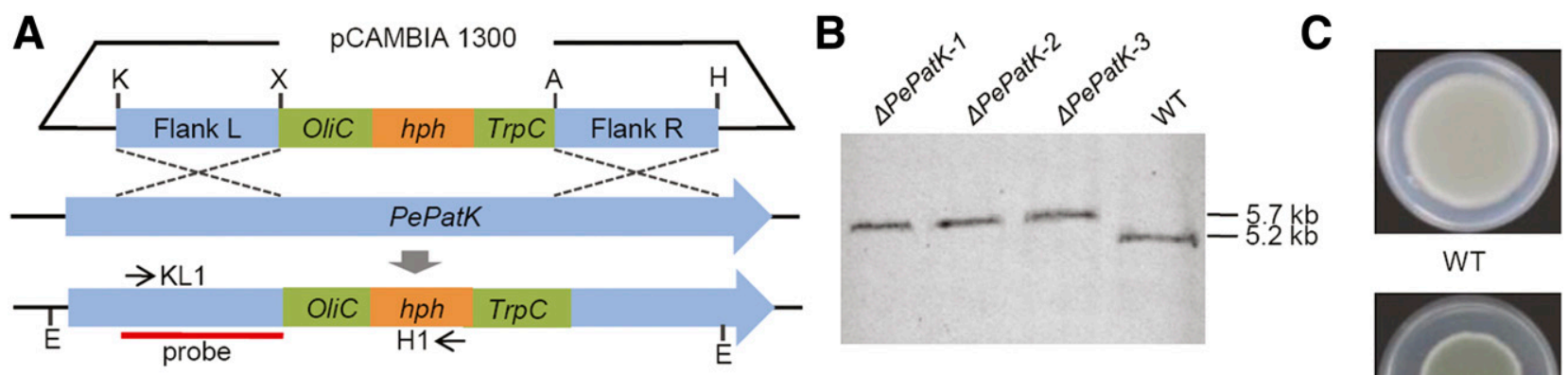

WT
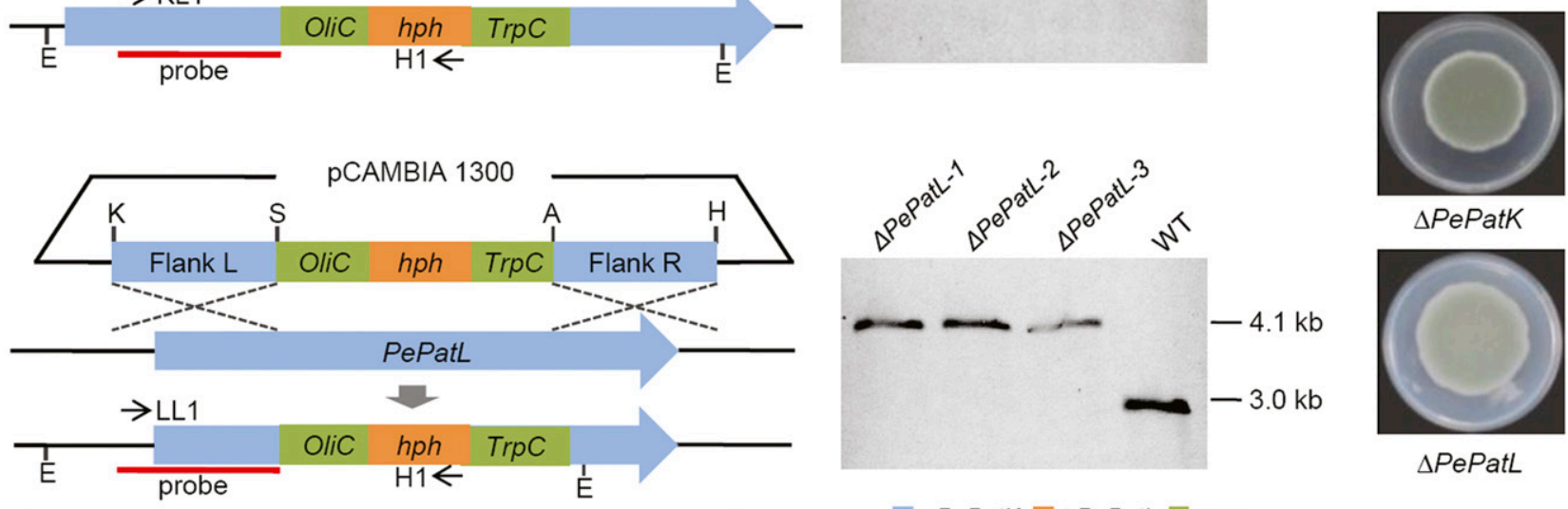

$\triangle P e P a t K$

D

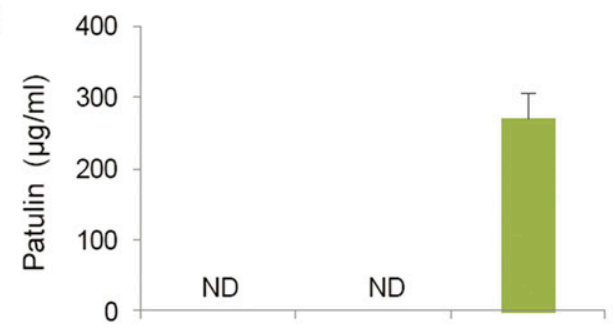

E

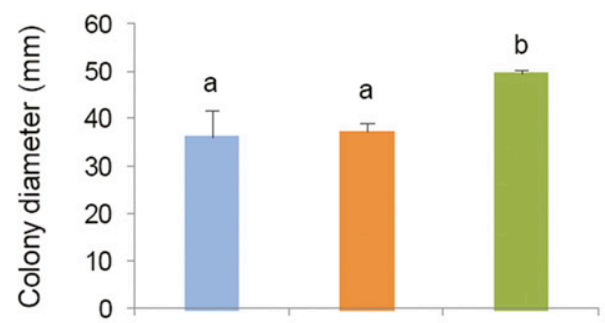

$\mathbf{F}$

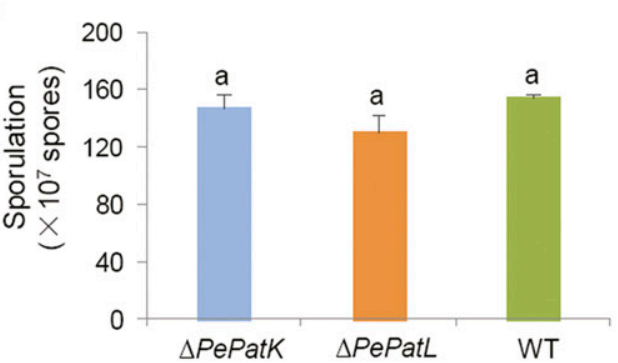

G $\quad 30$

${ }_{\Delta P e P a t K} \square{ }_{\Delta P e P a t L} \square \mathrm{WT}$

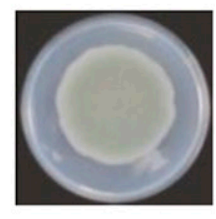

$\triangle P e P a t L$
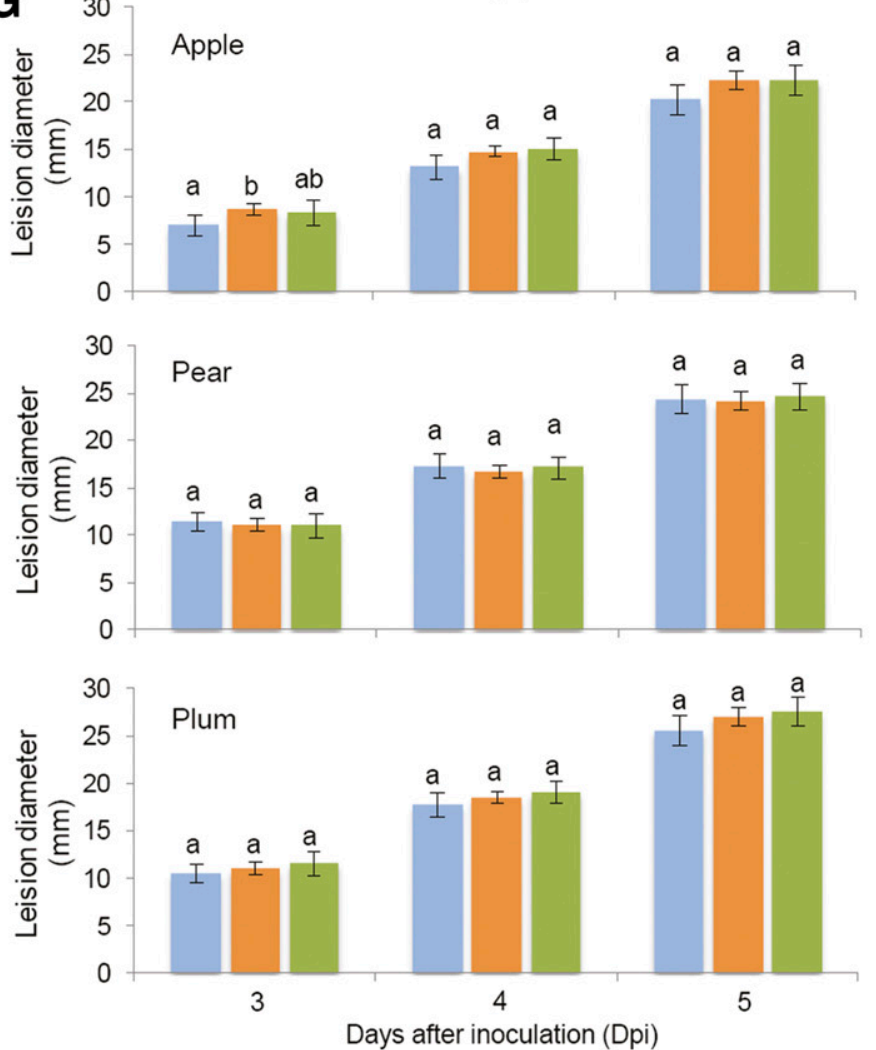

Fig. 7. Generation of PePatK and PePatL mutants and phenotype analysis. A, Replacement strategy for deletion of PePatK and PePatL. The gene replacement vector was constructed by cloning Flank $L$ and Flank $R$ on each side of the hygromycin resistance cassette of pCAMBIA 1300. Primer pairs KL1 and H1 and LL1 and H1 were used for flank-spanning polymerase chain reaction to verify the positive transformants. Probe $=$ digoxigenin-labeled Flank L for Southern blot analysis. Restriction sites: $\mathrm{A}=$ AscI $, \mathrm{E}=E c o \mathrm{RV}, \mathrm{H}=$ HindIII, $\mathrm{K}=K p n \mathrm{I}, \mathrm{S}=$ SalI, and $\mathrm{X}=\mathrm{XbaI}$. B, Southern blot analysis of the wild-type strain (WT) and three knockout mutants of each target gene. Genomic DNA was digested with EcoRV and was hybridized with a probe (digoxigenin-labeled Flank L). C, Colony morphology of WT and knockout mutants. D, Patulin production in WT and knockout mutants after being cultured for 10 days on potato dextrose agar (PDA). ND means no patulin production was detected. E and F, Colony diameter and sporulation of WT and knockout mutants incubated on PDA plates at $25^{\circ} \mathrm{C}$ for 10 days. G, Lesion diameters after 3,4 , or 5 days in apple, pear, and plum fruit inoculated with either WT, $\triangle P e P a t K-1$, or $\triangle P e P a t L-3$ knockout mutants and kept at $25^{\circ} \mathrm{C}$. Error bars represent standard deviation of three independent biological replicates. Columns with different letters are significantly different from each other, using the least significant difference test $(P<0.05)$. 
Saccharomyces cerevisiae and AFLR in A. flavus and A. nidulans. In this regard, PePatL was found to possess a $\mathrm{Zn}$ $(\mathrm{II})_{2} \mathrm{Cys}_{6}$ binuclear cluster DNA binding motif at the Nterminal end of the protein (Fig. 8A). The expression of genes in the patulin cluster in both the $\triangle P e P a t L$ mutant and the wildtype strain was compared. The results indicated that the examined genes in the patulin cluster were only marginally expressed in the $\triangle P e P a t L$ mutant when it was grown under patulin permissive condition (Fig. 8B). Additionally, microscopic analysis of a PePatL-green fluorescent protein (GFP) fusion protein indicated that PePatL is localized in the nuclei of P. expansum (Fig. 8C).

\section{DISCUSSION}

Some fungal plant pathogens cause economic losses not only by decaying the plant material but also by producing harmful mycotoxins (Calvo et al. 2002). P. expansum is able to infect a great number of fruit hosts and produces patulin in the contaminated tissues (Andersen et al. 2004). In contrast, P. italicum and $P$. digitatum only infect citrus fruit but do not produce patulin (Marcet-Houben et al. 2012). The molecular basis of host specificity and patulin biosynthesis of Penicillium species, however, has not been elucidated. Bioinformatic analysis on the genome sequence of a fungal pathogen can be used to assist the discovery of molecular mechanisms that are responsible for pathogenicity and the production of SM (Fierro et al. 1995; Marcet-Houben et al. 2012).

In the present study, the genomes of both $P$. expansum and $P$. italicum were sequenced (Fig. 1) and a high colinearity was found between the two species (Fig. 1E). The numbers of proteins in various functional categories, however, are greater in $P$. expansum than in P. italicum (Figs. 2, 3, and 4). In particular, $P$. expansum contains more genes encoding CAZymes and secreted proteases than either P. italicum or P. digitatum (Fig. 3A to F). CAZymes in plant pathogens are responsible for the synthesis, degradation, and modification of carbohydrates and, thus, play an important role in the breakdown of plant cell walls and in host-pathogen interactions (Zhao et al. 2014; Zheng et al. 2013). Fungi secrete enzymes to break down biopolymers from hosts and utilize them as nutrients (Marcet-Houben et al. 2012).
Secreted proteins from pathogens may also determine the progress and success of an infection (Li et al. 2012; Mueller et al. 2008; Qin et al. 2007). Therefore, the larger number of CAZymerelated genes in $P$. expansum may be partially responsible for the wider range of fruit hosts, relative to $P$. italicum and $P$. digitatum.

Several fungal pathogens produce bioactive small molecules, commonly classified as SM, that play a significant role in the development and life cycle of fungi (Keller et al. 2005). During the infection process, fungal pathogens may produce abundant levels of SM in plant hosts (Möbius and Hertweck 2009). SM genes in fungi are typically organized in clusters around backbone genes that encode synthases, such as PKS, NRPS, DMATS, and PKS-NPRS hybrids (Andersen et al. 2013). In this study, $P$. expansum was found to possess twice as many of these backbone genes than $P$. italicum and $P$. digitatum (Fig. 4) and more SM gene clusters (Fig. 5). The larger number and variety of SM gene clusters present in P. expansum (Fig. 5) may possibly contribute toward its wide host range.

In the present study, a cluster of 15 genes, putatively responsible for patulin biosynthesis in P. expansum, was identified (Fig. 5C; Table 2), which is the same number of genes as a putative patulin cluster in A. clavatus (Artigot et al. 2009). All 15 genes in the cluster were up-regulated when $P$. expansum was cultured under static (patulin-permissive) conditions (Fig. 6A and C), suggesting that all of the genes may be potentially related to patulin biosynthesis in P. expansum. A total of 1,642 upregulated and 1,392 downregulated genes were detected by transcriptome analysis when $P$. expansum was cultured under static conditions (Fig. 6A), including the SM backbone genes and genes encoding CYP450s, $\mathrm{ABC}$ transporters, and MFS transporters (Fig. 6B). CYP450s facilitate a wide variety of biochemical reactions in microorganisms and are critical for SM production (Kelly and Kelly 2013). MFS and $\mathrm{ABC}$ transporters are the two biggest families of fungal transporters and mediate transport of intermediates and export toxic end products in the secondary metabolism pathway (Del Sorbo et al. 2000). The differential expression of many of these genes in P. expansum grown under static versus shaking conditions indicated that culture condition has a significant effect on SM profiles in this fungal pathogen.

Using an Agrobacterium-mediated transformation method, two genes in the patulin cluster, PePatK and PePatL, were deleted.
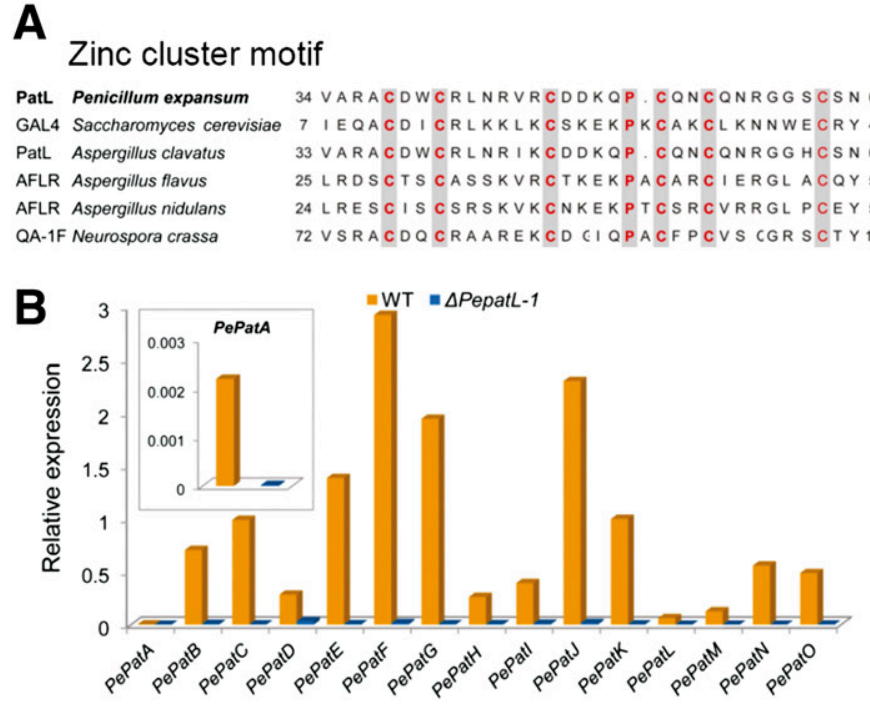
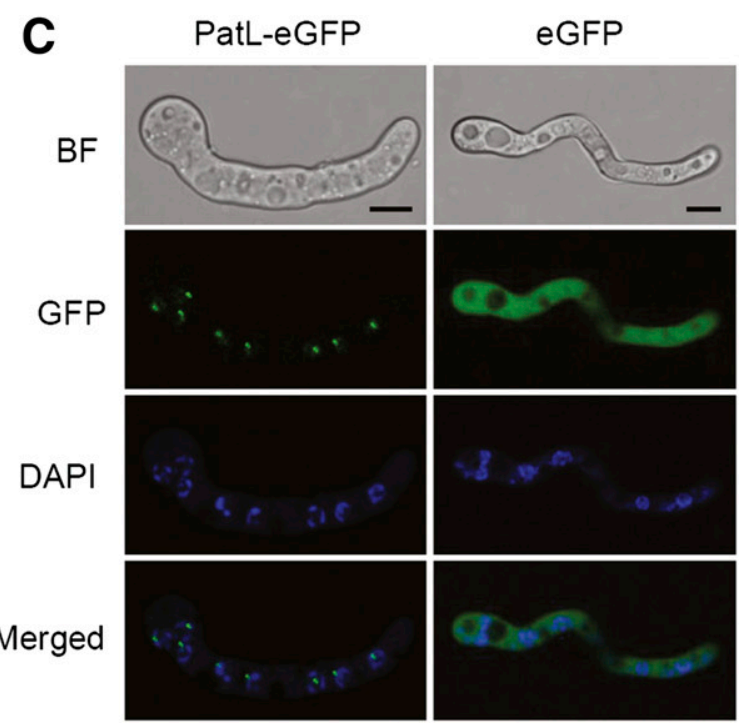

Fig. 8. Characterization of PePatL, a putative transcription factor in the patulin gene cluster of Penicillium expansum. A, PePatL possesses a conserved zinc cluster motif present in other demonstrated or putative transcription factors. B, Reverse transcription quantitative polymerase chain reaction analysis of the expression of patulin cluster genes in wild-type (WT) and the PePatL knockout mutant of P. expansum cultured under static condition for 4 d. C, Subcellular localization of PePatL. A transformant carrying the empty vector expressing only enhanced green fluorescent protein (eGFP) was used as a control. PePatL tagged with eGFP is localized in nuclei as determined with the aid of a specific nuclear stain, $4^{\prime}, 6$-diamidino-2-phenylindole. Bar $=5 \mu$ m. 
These deletions resulted in a complete inhibition of patulin production in the P. expansum knockout mutants (Fig. 7D). 6-MSAS, encoded by $P a t K$, has been reported to catalyze the first step of patulin biosynthesis, which involves the conversion of acetyl CoA and malonyl-CoA to 6-methylsalicylic acid in other Penicillium species (Puel et al. 2010). Our results demonstrated that PePatK is also crucial for the biosynthesis of patulin in P. expansum. PePatL has a conserved $\mathrm{Zn}(\mathrm{II})_{2} \mathrm{Cys}_{6}$ binuclear cluster DNA binding motif (Fig. 8A) and is similar to AFLR, a demonstrated transcription factor associated with the aflatoxin biosynthetic pathway (Yu et al. 2004). PePatL was observed to be localized in the nuclei of P. expansum (Fig. 8C) and affected the expression of other genes in the patulin cluster (Fig. 8B). These data support the premise that PePatL may act as a specific transcription factor in the patulin biosynthetic pathway. Importantly, a loss of the ability to produce patulin in the $\triangle P e P a t K$ and $\triangle$ PePatL mutants did not impair virulence (Fig. $7 \mathrm{G}$ ), indicating that patulin does not act as a pathogenicity factor or contribute to the virulence of $P$. expansum. In fact, most patulin-producing fungal species are not plant pathogens. Since patulin can exhibit antibiotic activity against some bacteria and fungi (Moake et al. 2005), the biological significance of patulin production in $P$. expansum may be to improve the ability of $P$. expansum to compete with other microbial organisms in specific environmental niches.

In summary, this study provides information on the molecular basis of pathogenicity and patulin biosynthesis in three Penicillium species based on the analysis of the sequenced genome of $P$. expansum and $P$. italicum. Most importantly we provide (i) the genome sequence of both $P$. expansum and P. italicum; (ii) an analysis of the composition of each genome and of important pathogenicity-related characteristics in three Penicillium species; (iii) prediction of 55 gene clusters potentially related to secondary metabolism and identification of a cluster of 15 genes that may be involved in patulin biosynthetic pathway in P. expansum; and (iv) determination of a crucial role of PePatL and PePatK in the patulin biosynthetic pathway and a demonstration that patulin production does not play a role in the virulence of $P$. expansum. We observed that $P$. expansum contains more pathogenic-related genes and SM gene clusters, in particular an intact patulin cluster, than $P$. italicum or $P$. digitatum. The obtained genome sequence data also provides information needed to further explore the molecular basis for other biological characteristics in Penicillium species.

\section{MATERIALS AND METHODS}

\section{Fungal strains.}

$P$. expansum $\mathrm{T} 01$ and $P$. italicum B3 were isolated from infected apple and sweet orange fruits, respectively, that exhibited typical blue mold symptoms. Two strains were purified by single-spore isolation and were stored at $-80^{\circ} \mathrm{C}$ prior to use.

\section{Extraction of genomic DNA and total RNA for sequencing.}

$P$. expansum $\mathrm{T} 01$ and $P$. italicum $\mathrm{B} 3$ were cultured on potato dextrose agar (PDA) plates. After 7 to 10 days, the spores were harvested by flooding with sterile distilled water. The concentration of the spore suspension was adjusted with a hemocytometer to $1 \times 10^{7}$ spores per milliliter.

For genome sequencing, a 1-ml aliquot of a spore suspension was added to $20 \mathrm{ml}$ Czapek yeast extract (CY) medium ( $3 \mathrm{~g}$ of $\mathrm{NaNO}_{3}, 1 \mathrm{~g}$ of $\mathrm{K}_{2} \mathrm{HPO}_{4} \cdot 3 \mathrm{H}_{2} \mathrm{O}, 0.5 \mathrm{~g}$ of $\mathrm{KCl}, 0.5 \mathrm{~g}$ of $\mathrm{MgSO}_{4} \cdot 7 \mathrm{H}_{2} \mathrm{O}, 0.01 \mathrm{~g}$ of $\mathrm{FeSO}_{4} \cdot 7 \mathrm{H}_{2} \mathrm{O}, 30 \mathrm{~g}$ of sucrose, $5 \mathrm{~g}$ of yeast extract, 1 liter of distilled water, $\mathrm{pH}$ 5.2) in 50-ml conical flasks and was cultured at $25^{\circ} \mathrm{C}$ on a rotary shaker at $200 \mathrm{rpm}$ for 3 days. Mycelium was collected by filtering the liquid culture through four layers of cheesecloth. The obtained mycelium was then washed thoroughly with cold sterile distilled water. A 2.0-g (wet weight) aliquot of mycelium was used for genomic DNA extraction with a DNeasy plant mini kit (Qiagen, Valencia, CA, U.S.A.).

Total RNA was extracted from four different samples of $P$. expansum and $P$. italicum, respectively. Sample I: spores collected from PDA plates cultured for 7 days at $25^{\circ} \mathrm{C}$; Sample II: spores incubated in liquid CY medium for $13 \mathrm{~h}$ with shaking at 200 rpm; sample III: mycelium grown in liquid CY medium for 4 days with shaking at $200 \mathrm{rpm}$; and sample IV: mycelium grown in liquid CY medium for 4 days without shaking. Total RNA was isolated from 0.2 -g (wet weight) aliquots of each sample, using TRIzol. RNA from each sample was pooled equally before sequencing. The RNA library was constructed using an Illumina TruSeq RNA sample preparation kit and was sequenced on Hiseq2000 with the pair-end mode. RNA-seq data were collected for improvement of gene prediction.

\section{Genome sequencing and assembly.}

The genome of $P$. expansum T01 and $P$. italicum B3 was sequenced with an Illumina Hiseq2000, using a whole-genome shotgun-sequencing strategy. Four sequencing libraries were constructed for $P$. expansum, with insert sizes of 180, 300, 3, and $8 \mathrm{~kb}$. Illumina TruSeq DNA sample preparation kits were used for the construction of the two pair-end libraries (180 and $300 \mathrm{bp}$ ). The two large-insert size libraries ( 3 and $8 \mathrm{~kb}$ ) were constructed using the Roche mate-pair library preparation method. A 21.6-Gb reads sequence was generated by Hiseq2000, using 100-bp pairend mode, representing approximately 527× genome coverage. Clean reads from the two libraries with an insert size of 180 and 300 bp were assembled using SOAPdenovo (V1.05) assembler (Li et al. 2008) to construct scaffolds. Then, using the clean reads from the large-insert size libraries of 3 and $8 \mathrm{~kb}$, the preassembled scaffolds were processed, scaffolding again using the stand-alone scaffolding tool SSPACE (V2.0) (Boetzer et al. 2011). Last, SOAP GapCloser (V1.12) (Li et al. 2009) was used to fill gaps in the obtained scaffolds. Three sequencing libraries were constructed for $P$. italicum, with insert sizes of 180,300 , and $500 \mathrm{bp}$, respectively. A t 5.7-Gb reads sequence was generated with 100-bp pair end, representing approximately $193 \times$ genome coverage.

\section{Gene annotation.}

For gene prediction, a combination of three methods was used. First, homology-based gene prediction was performed using Exonerate (v2.2.0) (Slater and Birney 2005), and then, Augustus (v2.5.5) (Stanke et al. 2004), Glimmer-HMM (v3.0.2) (Majoros et al. 2004), and GeneMark.hmm (v2.3) (Lukashin and Borodovsky 1998) were used to obtain ab initio prediction of gene structure. Last, RNA-Seq data were de novo assembled into transcripts by Trinity (Grabherr et al. 2011) to improve predicted gene structure. All of the above data were combined by EVM (Haas et al. 2008 ) to produce a consensus gene set. Then, PASA (Haas et al. 2003) was used to update the EVM gene structures and add untranslated region annotations. For functional annotation, protein sequences were blasted against a series of protein databases, including Swiss-Prot, TrEMBL, NCBI nr (National Center for Biotechnology Information nonredundant), and KOG databases, and the best hit was used to infer their biological function. GO annotation was performed using Blast2GO, which assigned homologous sequences aligned by BLAST with Uniprot and the NCBI nr database to GO terms.

\section{Annotation of specific gene categories.}

The SM unknown region finder (SMURF) (Khaldi et al. 2010) was used to predict backbone genes and SM gene clusters. The Fungal Cytochrome P450 database was used to identify cytochrome P450 enzymes by BLAST (blastp), with an e-value cutoff of $1 \mathrm{e}-5$ and alignment coverage $\geq 80 \%$. Transporters were 
identified based on BLAST searches (e value $\leq 1 \mathrm{e}-10$ and $>35$ identity of protein sequence) against the Transporter Classification database and the NCBI protein sequence database. The CAZyme genes were identified using CAT (Park et al. 2010). SignalP (v4.1) (Petersen et al. 2011) was used to predict secreted proteins. A MEROPS Batch BLAST analysis (Rawlings et al. 2010) was performed to identify secreted peptidase genes.

\section{Transposable element analysis.}

To identify the repetitive sequence in the genome, Repeatmasker was used with the Repbase library (v17.11) and the species option of 'Fungi'. The repeat sequence was masked with 'N'.

\section{Genome synteny analysis.}

The collinearity between $P$. expansum and $P$. italicum genomes was analyzed using SyMAP (v4.0) (Soderlund et al. 2011). The longest eight scaffolds of $P$. expansum, representing $98 \%$ of the assembled genome, were used in the analysis. The $P$. italicum genome was aligned to the eight scaffolds of $P$. expansum. The $P$. italicum scaffold sequences were merged together based on the alignment results and were named Pi_1 to $\mathrm{Pi} 8$. The collinearity between the $P$. expansum and $P$. italicum genomes was then analyzed using SyMAP.

\section{Gene family analysis.}

Gene families were generated using MCL software (Version 12-068) (Enright et al. 2002), which utilizes a Markov clustering algorithm to cluster proteins into families based on precomputed sequence similarity information among all genes in all taxa. An all-vs-all BLAST search of each species' protein was performed, and the blastp results were filtered using threshold limits of e values $\leq 1 \mathrm{e}-5$, alignment identity $\geq 30 \%$, and an alignment coverage $\geq 50 \%$. Gene families were generated by MCL software using an inflation value of 2.0.

\section{Phylogenetic analysis.}

A whole-genome cladogram displaying the phylogenetic relationships between $P$. expansum, $P$. italicum, and $P$. digitatum was constructed using CVTree with Talaromyces marneffe $i$ as an outgroup (O'Connell et al. 2012). The genome sequences of $P$. digitatum PHI26 and Talaromyces marneffei PM1 were downloaded from NCBI under accessions AKCT00000000 (Marcet-Houben et al. 2012) and AGCC00000000 (Woo et al. 2011), respectively. Phylogenetic trees of PKS, NRPS, DMAT, and hybrids were constructed using the maximum-likelihood approach implemented in PhyML (Guindon et al. 2010).

\section{Expression analysis of GH78 family genes induced by apple and citrus pectin.}

For expression analysis of genes in the GH78 family, RT-qPCR analysis was performed on an ABI StepOnePlus real-time PCR system (Applied Biosystems, Foster City, CA, U.S.A.). A spore suspension of $P$. expansum was added to $20 \mathrm{ml}$ of pectin-CY medium (CY without sucrose and with $1 \%$ pectin from apple or citrus) to obtain a final concentration of $10^{6}$ spores pere milliliter and were cultured at $25^{\circ} \mathrm{C}$ with shaking at $180 \mathrm{rpm}$. Mycelium was harvested using four layers of cheesecloth at 24 and $48 \mathrm{~h}$ after inoculation. Total RNA was isolated from the mycelium of $P$. expansum as described above. RT-qPCR was carried out in a total volume of $20 \mu \mathrm{l}$, containing $10 \mu \mathrm{l}$ of $2 \times$ Ultra SYBR mixture (with high ROX) (CWBIO Co Ltd., Beijing), $2 \mu$ of 10x diluted cDNA, $0.4 \mu \mathrm{l}$ of each primer $(10 \mathrm{mM})$, and $7.2 \mu \mathrm{l}$ of RNase-free water. The primers used for amplification are listed in Supplementary Table S9. Transcript levels were normalized against the $\beta$-tublin gene (GenBank accession number AF003248.1), and relative expression levels were calculated using the $2^{-\Delta \Delta C T}$ method (Livak and Schmittgen 2001).
Patulin production and gene expression analysis under different cultural conditions.

Spores of $P$. expansum were inoculated in liquid CY medium as described above, and were cultured under shaking (200 rpm) or static conditions at $25^{\circ} \mathrm{C}$. After 2 and 4 days, the mycelium was harvested and quickly frozen with liquid nitrogen for RNA extraction. CY medium filtrates were filtered through $0.45-\mu \mathrm{m}$ filters before being analyzed by HPLC for patulin production. The HPLC system was equipped with an auto sampler (Waters 2707, Waters Corp., Milford, MA, U.S.A.), binary HPLC pump (Waters 1525), and a UV/visible detector (Waters 2487). A C18 column $(5 \mu \mathrm{m}, 250 \times 4.6 \mathrm{~mm}$, Intersil ODS-3; GL Sciences, Tokyo) was used for separation at $30^{\circ} \mathrm{C}$. A mixture of water and acetonitrile $(90: 10, \mathrm{vol} / \mathrm{vol})$ at a flow rate of $1 \mathrm{ml} / \mathrm{min}$ was used as the mobile phase in an isocratic elution mode. Detection of patulin was performed at a wavelength of $276 \mathrm{~nm}$.

For transcriptome analysis, total RNA was extracted from the mycelium of cultures. Two independent biological samples from each culture condition were used. The RNA library was constructed using the method described above and was sequenced on a Hiseq2500 with the pair-end mode. About 2-Gb reads sequence was generated for each sample. After removing sequencing adapters and trimming consecutive low-quality bases (Quality < 20) from both the 5' and 3' ends of the reads, high-quality RNA-seq reads from $P$. expansum were aligned to the reference genome using Tophat2 (v2.0.11) (Kim et al. 2013) with default parameters. Cufflinks (Trapnell et al. 2010) was then used to quantify expression. Abundances are reported as normalized fragments per kilobase of transcript per million mapped reads (FPKM). Differential expression of a gene is considered to be significant if its expression differs between two samples at a $P$ value $\leq 0.001$, as calculated by Cufflinks.

For expression analysis of genes in the patulin cluster, total RNA was isolated from the mycelium of $P$. expansum cultured for 2 and 4 days. RT-qPCR was carried out as described above. The primers used for amplification are listed in Supplementary Table S10.

\section{Construction of the replacement vectors and transformation.}

The pLOB 7 and pCAMBIA 1300 vectors were provided by J. A. L. van Kan, Wageningen University, The Netherlands and Z. Ma, Zhejiang University, Hangzhou City, China, respectively. First, an EcoRI-HindIII DNA fragment containing the hygromycin phosphotransferase gene $h p h$ under control of the oliC promoter and $\operatorname{trpC}$ terminator was excised from $\mathrm{pLOB} 7$ and cloned into pCAMBIA 1300 to yield the binary vector pCHPH. For the construction of gene replacement vectors, two fragments flanking each of the target sequences were amplified and cloned into pCHPH up and down stream of $h p h$ to yield the vectors pCHPH-PatK and pCHPH-PatL, respectively. Primers for amplification of the flanking fragments are listed in Supplementary Table S11. The gene replacement vector was transformed into Agrobacterium tumefaciens EHA 105 by electroporation. The transformed $A$. tumefaciens strain was cocultured with $P$. expansum spores on a cellophane sheet that was placed on agar plates containing cocultivation medium $\left(10 \mathrm{mM} \mathrm{K} 2 \mathrm{HPO}_{4} \cdot 3 \mathrm{H}_{2} \mathrm{O}, 10 \mathrm{mM}\right.$ $\mathrm{KH}_{2} \mathrm{PO}_{4}, 2.5 \mathrm{mM} \mathrm{NaCl}, 2 \mathrm{mM} \mathrm{MgSO} \cdot 7 \mathrm{H}_{2} \mathrm{O}, 0.45 \mathrm{mM} \mathrm{CaCl}$, $9 \mu \mathrm{M} \mathrm{FeSO} \cdot 7 \mathrm{H}_{2} \mathrm{O}, 4 \mathrm{mM}\left(\mathrm{NH}_{4}\right)_{2} \mathrm{SO}_{4}, 5 \mathrm{mM}$ glucose, $0.5 \%$ glycerol, $40 \mathrm{mM}$ MES, $200 \mu \mathrm{M}$ acetosyringone, and $1.5 \%$ agar, $\mathrm{pH}$ 5.3). After cocultivation, the cellophane sheet was transferred to CYA medium (CY added to $1.5 \%$ agar) that was supplemented with $250 \mu \mathrm{g}$ hygromycin B per millimeter to select transformants. Stability of the antibiotic-resistant phenotype was tested by repeated transfer of the putative transformants to fresh selection plates for three times. The resistant strains were verified by PCR, using primer pairs KL1 and $\mathrm{H} 1$ for PePatK and LL1 and 
H1 for PePatL. Then, the positive transformants were purified by single-spore isolation and were confirmed by Southern blot analysis to exclude transformants with ectopic integration sites. Genomic DNA for PCR confirmation and Southern blot analysis was isolated following the procedure of Raeder and Broda (1985). For Southern blot analysis, $10 \mu \mathrm{g}$ of genomic DNA was completely digested with EcoRV. The digests were fractionated by electrophoresis on an $0.8 \%$ agarose gel and were transferred to a Hybond-N+ nylon membrane (Mylab, Beijing) using alkaline transfer buffer $(0.4 \mathrm{M} \mathrm{NaOH}$ and $1 \mathrm{M} \mathrm{NaCl})$. After being washed in neutralization buffer $(0.5 \mathrm{M}$ Tris- $\mathrm{HCl}$ and $1 \mathrm{M} \mathrm{NaCl}, \mathrm{pH} 7.2$ ) and heated at $80^{\circ} \mathrm{C}$ for $1 \mathrm{~h}$, the membrane was hybridized and detected according to the instructions of the Dig hybridization detection kit (Mylab). The probes were labeled with digoxigenin by PCR, using the primers KL1 and KL2 and LL1 and LL2.

\section{Phenotype analysis of gene knockout mutants.}

Analysis of patulin production, colony growth, and sporulation were determined according to the methods of Sanzani et al. (2012) with some modifications. Five-microliter aliquots of a spore suspension with a concentration of $10^{5}$ spores per milliliter were inoculated in the center of 9-cm PDA plates and were incubated at $25^{\circ} \mathrm{C}$ in the dark for 10 days. Colony morphology, colony diameter, spore, and patulin production were then recorded. Patulin was extracted using acidified, distilled water and was determined using the HPLC system described above. For the pathogenicity assay, conidia of mutants $\triangle P e P a t K-1$ and $\triangle P e P a t L-3$ mutants and the wild-type strain of $P$. expansum were inoculated in wounded apple (Malus domestica cv. Fuji), pear (Pyrus pyrifolia cv. Huangguan), and plum (Prunus americana) fruit. Four wounds $(3 \times 3 \mathrm{~mm})$ were made with a sterile nail on the equator of each apple or pear fruit and two wounds $(3 \times 3 \mathrm{~mm})$ were made on each plum fruit. A $10-\mu l$ spore suspension $\left(1 \times 10^{4}\right.$ spores per milliliter $)$ was pipetted into each wound. Inoculated fruits were stored at $25^{\circ} \mathrm{C}$ for 5 days and lesion diameters were determined daily.

\section{ACKNOWLEDGMENTS}

We thank N. P. Keller and M. Long for helpful comments on the manuscript. We also thank J. A. L. van Kan and Z. Ma for supplying vectors. This work was supported by the National High Technology Research (863) Program of China (2012AA101607), National Natural Science Foundation of China (31030051), and the Chinese Academy of Sciences (KSCX2-EW-G-6). The funders had no role in study design, data collection and analysis, decision to publish, or preparation of the manuscript.

\section{LITERATURE CITED}

Amselem, J., Cuomo, C. A., van Kan, J. A., Viaud, M., Benito, E. P., Couloux, A., Coutinho, P. M., de Vries, R. P., Dyer, P. S., Fillinger, S., Fournier, E., Gout, L., Hahn, M., Kohn, L., Lapalu, N., Plummer, K. M., Pradier, J. M., Quévillon, E., Sharon, A., Simon, A., ten Have, A., Tudzynski, B., Tudzynski, P., Wincker, P., Andrew, M., Anthouard, V., Beever, R. E., Beffa, R., Benoit, I., Bouzid, O., Brault, B., Chen, Z., Choquer, M., Collémare, J., Cotton, P., Danchin, E. G., Da Silva, C., Gautier, A., Giraud, C., Giraud, T., Gonzalez, C., Grossetete, S., Güldener, U., Henrissat, B., Howlett, B. J., Kodira, C., Kretschmer, M., Lappartient, A., Leroch, M., Levis, C., Mauceli, E., Neuvéglise, C., Oeser, B., Pearson, M., Poulain, J., Poussereau, N., Quesneville, H., Rascle, C., Schumacher, J., Ségurens, B., Sexton, A., Silva, E., Sirven, C., Soanes, D. M., Talbot, N. J., Templeton, M., Yandava, C., Yarden, O., Zeng, Q., Rollins, J. A., Lebrun, M. H., and Dickman, M. 2011. Genomic analysis of the necrotrophic fungal pathogens Sclerotinia sclerotiorum and Botrytis cinerea. PLoS Genet. 7:e1002230.

Andersen, B., Smedsgaard, J., and Frisvad, J. C. 2004. Penicillium expansum: consistent production of patulin, chaetoglobosins, and other secondary metabolites in culture and their natural occurrence in fruit products. J. Agric. Food Chem. 52:2421-2428.
Andersen, M. R., Nielsen, J. B., Klitgaard, A., Petersen, L. M., Zachariasen, M., Hansen, T. J., Blicher, L. H., Gotfredsen, C. H., Larsen, T. O., Nielsen, K. F., and Mortensen, U. H. 2013. Accurate prediction of secondary metabolite gene clusters in filamentous fungi. Proc. Natl. Acad. Sci. U.S.A. 110:E99-E107.

Artigot, M. P., Loiseau, N., Laffitte, J., Mas-Reguieg, L., Tadrist, S., Oswald, I. P., and Puel, O. 2009. Molecular cloning and functional characterization of two CYP619 cytochrome P450s involved in biosynthesis of patulin in Aspergillus clavatus. Microbiology 155 (Pt 5):1738-1747.

Bayram, O., Krappmann, S., Ni, M., Bok, J. W., Helmstaedt, K., Valerius, O., Braus-Stromeyer, S., Kwon, N. J., Keller, N. P., Yu, J. H., and Braus, G. H. 2008. VelB/VeA/LaeA complex coordinates light signal with fungal development and secondary metabolism. Science 320:1504-1506.

Bennett, J. W., and Klich, M. 2003. Mycotoxins. Clin. Microbiol. Rev. 16: 497-516.

Blackman, L. M., Cullerne, D. P., and Hardham, A. R. 2014. Bioinformatic characterisation of genes encoding cell wall degrading enzymes in the Phytophthora parasitica genome. BMC Genomics 15:785.

Boetzer, M., Henkel, C. V., Jansen, H. J., Butler, D., and Pirovano, W. 2011. Scaffolding pre-assembled contigs using SSPACE. Bioinformatics 27:578-579.

Bok, J. W., and Keller, N. P. 2004. LaeA, a regulator of secondary metabolism in Aspergillus spp. Eukaryot. Cell 3:527-535.

Calvo, A. M., Wilson, R. A., Bok, J. W., and Keller, N. P. 2002. Relationship between secondary metabolism and fungal development. Microbiol. Mol. Biol. Rev. 66:447-459.

Cheeseman, K., Ropars, J., Renault, P., Dupont, J., Gouzy, J., Branca, A., Abraham, A. L., Ceppi, M., Conseiller, E., Debuchy, R., Malagnac, F., Goarin, A., Silar, P., Lacoste, S., Sallet, E., Bensimon, A., Giraud, T., and Brygoo, Y. 2014. Multiple recent horizontal transfers of a large genomic region in cheese making fungi. Nat. Commun. 5:2876.

Del Sorbo, G., Schoonbeek, H., and De Waard, M. A. 2000. Fungal transporters involved in efflux of natural toxic compounds and fungicides. Fungal Genet. Biol. 30:1-15.

Dunwell, J. M., Khuri, S., and Gane, P. J. 2000. Microbial relatives of the seed storage proteins of higher plants: conservation of structure and diversification of function during evolution of the cupin superfamily. Microbiol. Mol. Biol. Rev. 64:153-179.

Enright, A. J., Van Dongen, S., and Ouzounis, C. A. 2002. An efficient algorithm for large-scale detection of protein families. Nucleic Acids Res. 30:1575-1584.

Fierro, F., Barredo, J. L., Díez, B., Gutierrez, S., Fernández, F. J., and Martín, J. F. 1995. The penicillin gene cluster is amplified in tandem repeats linked by conserved hexanucleotide sequences. Proc. Natl. Acad. Sci. U.S.A. 92:6200-6204.

Grabherr, M. G., Haas, B. J., Yassour, M., Levin, J. Z., Thompson, D. A., Amit, I., Adiconis, X., Fan, L., Raychowdhury, R., Zeng, Q., Chen, Z., Mauceli, E., Hacohen, N., Gnirke, A., Rhind, N., di Palma, F., Birren, B. W., Nusbaum, C., Lindblad-Toh, K., Friedman, N., and Regev, A. 2011. Full-length transcriptome assembly from RNA-Seq data without a reference genome. Nat. Biotechnol. 29:644-652.

Guindon, S., Dufayard, J. F., Lefort, V., Anisimova, M., Hordijk, W., and Gascuel, O. 2010. New algorithms and methods to estimate maximumlikelihood phylogenies: assessing the performance of PhyML 3.0. Syst. Biol. 59:307-321.

Haas, B. J., Delcher, A. L., Mount, S. M., Wortman, J. R., Smith, R. K., Jr., Hannick, L. I., Maiti, R., Ronning, C. M., Rusch, D. B., Town, C. D., Salzberg, S. L., and White, O. 2003. Improving the Arabidopsis genome annotation using maximal transcript alignment assemblies. Nucleic Acids Res. 31:5654-5666.

Haas, B. J., Salzberg, S. L., Zhu, W., Pertea, M., Allen, J. E., Orvis, J., White, O., Buell, C. R., and Wortman, J. R. 2008. Automated eukaryotic gene structure annotation using EVidenceModeler and the Program to Assemble Spliced Alignments. Genome Biol. 9:R7.

Keller, N. P., Turner, G., and Bennett, J. W. 2005. Fungal secondary metabolism - from biochemistry to genomics. Nat. Rev. Microbiol. 3:937-947.

Kelly, S. L., and Kelly, D. E. 2013. Microbial cytochromes P450: Biodiversity and biotechnology. Where do cytochromes P450 come from, what do they do and what can they do for us? Philos. Trans. R. Soc. Lond. B Biol. Sci. 368:20120476.

Khaldi, N., Seifuddin, F. T., Turner, G., Haft, D., Nierman, W. C., Wolfe, K. H., and Fedorova, N. D. 2010. SMURF: Genomic mapping of fungal secondary metabolite clusters. Fungal Genet. Biol. 47:736-741.

Kim, D., Pertea, G., Trapnell, C., Pimentel, H., Kelley, R., and Salzberg, S. L. 2013. TopHat2: Accurate alignment of transcriptomes in the presence of insertions, deletions and gene fusions. Genome Biol. 14:R36.

Li, R., Li, Y., Kristiansen, K., and Wang, J. 2008. SOAP: Short oligonucleotide alignment program. Bioinformatics 24:713-714. 
Li, R., Yu, C., Li, Y., Lam, T. W., Yiu, S. M., Kristiansen, K., and Wang, J. 2009. SOAP2: An improved ultrafast tool for short read alignment. Bioinformatics 25:1966-1967.

Li, B. Q., Wang, W. H., Zong, Y. Y., Qin, G. Z., and Tian, S. P. 2012. Exploring pathogenic mechanisms of Botrytis cinerea secretome under different ambient $\mathrm{pH}$ based on comparative proteomic analysis of secretome. J. Proteome Res. 11:4249-4260.

Livak, K. J., and Schmittgen, T. D. 2001. Analysis of relative gene expression data using real-time quantitative PCR and the $2^{-\Delta \Delta C T}$ method. Methods 25:402-408.

Lombard, V., Golaconda Ramulu, H., Drula, E., Coutinho, P. M., and Henrissat, B. 2014. The carbohydrate-active enzymes database (CAZy) in 2013. Nucleic Acids Res. 42(Database issue):D490-D495.

Lukashin, A. V., and Borodovsky, M. 1998. GeneMark.hmm: New solutions for gene finding. Nucleic Acids Res. 26:1107-1115.

Majoros, W. H., Pertea, M., and Salzberg, S. L. 2004. TigrScan and GlimmerHMM: Two open source $a b$ initio eukaryotic gene-finders. Bioinformatics 20:2878-2879.

Marcet-Houben, M., Ballester, A. R., de la Fuente, B., Harries, E., Marcos, J. F., González-Candelas, L., and Gabaldón, T. 2012. Genome sequence of the necrotrophic fungus Penicillium digitatum, the main postharvest pathogen of citrus. BMC Genomics 13:646.

Moake, M. M., Padilla-Zakour, O. I., and Worobo, R. W. 2005. Comprehensive review of patulin control methods in foods. Compr. Rev. Food Sci. F. 4:8-21.

Möbius, N., and Hertweck, C. 2009. Fungal phytotoxins as mediators of virulence. Curr. Opin. Plant Biol. 12:390-398.

Mueller, O., Kahmann, R., Aguilar, G., Trejo-Aguilar, B., Wu, A., and de Vries, R. P. 2008. The secretome of the maize pathogen Ustilago maydis. Fungal Genet. Biol. 45 (Suppl 1):S63-S70.

O'Connell, R. J., Thon, M. R., Hacquard, S., Amyotte, S. G., Kleemann, J., Torres, M. F., Damm, U., Buiate, E. A., Epstein, L., Alkan, N., Altmüller, J., Alvarado-Balderrama, L., Bauser, C. A., Becker, C., Birren, B. W., Chen, Z., Choi, J., Crouch, J. A., Duvick, J. P., Farman, M. A., Gan, P., Heiman, D., Henrissat, B., Howard, R. J., Kabbage, M., Koch, C., Kracher, B., Kubo, Y., Law, A. D., Lebrun, M. H., Lee, Y. H., Miyara, I., Moore, N., Neumann, U., Nordström, K., Panaccione, D. G., Panstruga, R., Place, M., Proctor, R. H., Prusky, D., Rech, G., Reinhardt, R., Rollins, J. A., Rounsley, S., Schardl, C. L., Schwartz, D. C., Shenoy, N., Shirasu, K., Sikhakolli, U. R., Stüber, K., Sukno, S. A., Sweigard, J. A., Takano, Y., Takahara, H., Trail, F., van der Does, H. C., Voll, L. M., Will, I., Young, S., Zeng, Q., Zhang, J., Zhou, S., Dickman, M. B., Schulze-Lefert, P., Ver Loren van Themaat, E., Ma, L. J., and Vaillancourt, L. J. 2012. Lifestyle transitions in plant pathogenic Colletotrichum fungi deciphered by genome and transcriptome analyses. Nat. Genet. 44:1060-1065.

Park, B. H., Karpinets, T. V., Syed, M. H., Leuze, M. R., and Uberbacher, E. C. 2010. CAZymes analysis toolkit (CAT): Web service for searching and analyzing carbohydrate-active enzymes in a newly sequenced organism using CAZy database. Glycobiology 20:1574-1584.

Petersen, T. N., Brunak, S., von Heijne, G., and Nielsen, H. 2011. SignalP 4.0: discriminating signal peptides from transmembrane regions. Nat. Methods 8:785-786.

Pfeiffer, E., Gross, K., and Metzler, M. 1998. Aneuploidogenic and clastogenic potential of the mycotoxins citrinin and patulin. Carcinogenesis 19:1313-1318.

Puel, O., Galtier, P., and Oswald, I. P. 2010. Biosynthesis and toxicological effects of patulin. Toxins Basel 2:613-631.

Qin, G., Tian, S., Chan, Z., and Li, B. 2007. Crucial role of antioxidant proteins and hydrolytic enzymes in pathogenicity of Penicillium expansum: analysis based on proteomics approach. Mol. Cell. Proteomics 6:425-438.

Raeder, U., and Broda, P. 1985. Rapid preparation of DNA from filamentous fungi. Lett. Appl. Microbiol. 1:17-20.

Rawlings, N. D., Barrett, A. J., and Bateman, A. 2010. MEROPS: The peptidase database. Nucleic Acids Res. 38(Database issue):D227-D233.

Rouxel, T., Grandaubert, J., Hane, J. K., Hoede, C., van de Wouw, A. P., Couloux, A., Dominguez, V., Anthouard, V., Bally, P., Bourras, S., Cozijnsen, A. J., Ciuffetti, L. M., Degrave, A., Dilmaghani, A., Duret, L., Fudal, I., Goodwin, S. B., Gout, L., Glaser, N., Linglin, J., Kema, G. H., Lapalu, N., Lawrence, C. B., May, K., Meyer, M., Ollivier, B., Poulain, J., Schoch, C. L., Simon, A., Spatafora, J. W., Stachowiak, A., Turgeon, B. G., Tyler, B. M., Vincent, D., Weissenbach, J., Amselem, J., Quesneville, H., Oliver, R. P., Wincker, P., Balesdent, M. H., and Howlett, B. J. 2011. Effector diversification within compartments of the Leptosphaeria maculans genome affected by repeat-induced point mutations. Nat. Commun. 2:202.

Sanzani, S. M., Reverberi, M., Punelli, M., Ippolito, A., and Fanelli, C. 2012. Study on the role of patulin on pathogenicity and virulence of Penicillium expansum. Int. J. Food Microbiol. 153:323-331.
Slater, G. S., and Birney, E. 2005. Automated generation of heuristics for biological sequence comparison. BMC Bioinformatics 6:31.

Soderlund, C., Bomhoff, M., and Nelson, W. M. 2011. SyMAP v3.4: A turnkey synteny system with application to plant genomes. Nucleic Acids Res. 39:e68.

Stanke, M., Steinkamp, R., Waack, S., and Morgenstern, B. 2004. AUGUSTUS: A web server for gene finding in eukaryotes. Nucleic Acids Res. 32(Suppl 2):W309-W312.

Strauss, J., and Reyes-Dominguez, Y. 2011. Regulation of secondary metabolism by chromatin structure and epigenetic codes. Fungal Genet. Biol. 48:62-69.

Sultana, A., Kallio, P., Jansson, A., Wang, J. S., Niemi, J., Mäntsälä, P., and Schneider, G. 2004. Structure of the polyketide cyclase SnoaL reveals a novel mechanism for enzymatic aldol condensation. EMBO J. 23: 1911-1921.

Tannous, J., El Khoury, R., Snini, S. P., Lippi, Y., El Khoury, A., Atoui, A., Lteif, R., Oswald, I. P., and Puel, O. 2014. Sequencing, physical organization and kinetic expression of the patulin biosynthetic gene cluster from Penicillium expansum. Int. J. Food Microbiol. 189: 51-60.

Trapnell, C., Williams, B. A., Pertea, G., Mortazavi, A., Kwan, G., van Baren, M. J., Salzberg, S. L., Wold, B. J., and Pachter, L. 2010. Transcript assembly and quantification by RNA-Seq reveals unannotated transcripts and isoform switching during cell differentiation. Nat. Biotechnol. 28:511-515.

van den Berg, M. A., Albang, R., Albermann, K., Badger, J. H., Daran, J. M., Driessen, A. J., Garcia-Estrada, C., Fedorova, N. D., Harris, D. M., Heijne, W. H., Joardar, V., Kiel, J. A., Kovalchuk, A., Martín, J. F., Nierman, W. C., Nijland, J. G., Pronk, J. T., Roubos, J. A., van der Klei, I. J., van Peij, N. N., Veenhuis, M., von Döhren, H., Wagner, C., Wortman, J., and Bovenberg, R. A. 2008. Genome sequencing and analysis of the filamentous fungus Penicillium chrysogenum. Nat. Biotechnol. 26:1161-1168.

Wang, I. K., Reeves, C., and Gaucher, G. M. 1991. Isolation and sequencing of a genomic DNA clone containing the $3^{\prime}$ terminus of the 6methylsalicylic acid polyketide synthetase gene of Penicillium urticae. Can. J. Microbiol. 37:86-95.

Wang, X., Chen, Q. and Lü, X. 2014. Pectin extracted from apple pomace and citrus peel by subcritical water. Food Hydrocolloids 38:129-137.

Weng, J. K., Li, Y., Mo, H., and Chapple, C. 2012. Assembly of an evolutionarily new pathway for $\alpha$-pyrone biosynthesis in Arabidopsis. Science 337:960-964.

White, S., O'Callaghan, J., and Dobson, A. D. W. 2006. Cloning and molecular characterization of Penicillium expansum genes upregulated under conditions permissive for patulin biosynthesis. FEMS Microbiol. Lett. 255:17-26.

Woo, P. C., Lau, S. K., Liu, B., Cai, J. J., Chong, K. T., Tse, H., Kao, R. Y., Chan, C. M., Chow, W. N., and Yuen, K. Y. 2011. Draft genome sequence of Penicillium marneffei strain PM1. Eukaryot. Cell 10: 1740-1741.

Yoder, O. C., and Turgeon, B. G. 1996. Molecular genetic evaluation of fungal molecules for roles in pathogenesis to plants. J. Genet. 75: 425-440.

Yu, J. H., and Keller, N. 2005. Regulation of secondary metabolism in filamentous fungi. Annu. Rev. Phytopathol. 43:437-458.

Yu, J. H., Butchko, R. A., Fernandes, M., Keller, N. P., Leonard, T. J., and Adams, T. H. 1996. Conservation of structure and function of the aflatoxin regulatory gene aflR from Aspergillus nidulans and A. flavus. Curr. Genet. 29:549-555.

Yu, J., Chang, P. K., Ehrlich, K. C., Cary, J. W., Bhatnagar, D., Cleveland, T. E., Payne, G. A., Linz, J. E., Woloshuk, C. P., and Bennett, J. W. 2004. Clustered pathway genes in aflatoxin biosynthesis. Appl. Environ. Microbiol. 70:1253-1262.

Zhao, Z., Liu, H., Wang, C., and Xu, J. R. 2014. Correction: Comparative analysis of fungal genomes reveals different plant cell wall degrading capacity in fungi. BMC Genomics 15:6.

Zheng, A., Lin, R., Zhang, D., Qin, P., Xu, L., Ai, P., Ding, L., Wang, Y., Chen, Y., Liu, Y., Sun, Z., Feng, H., Liang, X., Fu, R., Tang, C., Li, Q., Zhang, J., Xie, Z., Deng, Q., Li, S., Wang, S., Zhu, J., Wang, L., Liu, H., and $\mathrm{Li}, \mathrm{P}$. 2013. The evolution and pathogenic mechanisms of the rice sheath blight pathogen. Nat. Commun. 4:1424.

\section{AUTHOR-RECOMMENDED INTERNET RESOURCES}

Carbohydrate-Active enZYmes database: www.cazy.org Fungal Cytochrome P450 database: p450.riceblast.snu.ac.kr Repeatmasker program: www.repeatmasker.org Transport Classification database (TCDB): www.tcdb.org 